\title{
Some Results on Best Proximity Points of Cyclic Contractions in Probabilistic Metric Spaces
}

\author{
Manuel De la Sen ${ }^{1}$ and Erdal Karapınar ${ }^{2,3}$ \\ ${ }^{1}$ Institute of Research and Development of Processes IIDP, Faculty of Science and Technology, University of the Basque Country, P.O. \\ Box 644 de Bilbao Barrio Sarriena, Leioa, 48940 Bizkaia, Spain \\ ${ }^{2}$ Department of Mathematics, ATILIM University, Incek, 06836 Ankara, Turkey \\ ${ }^{3}$ Nonlinear Analysis and Applied Mathematics Research Group (NAAM), King Abdulaziz University, Jeddah 21589, Saudi Arabia
}

Correspondence should be addressed to Manuel De la Sen; manuel.delasen@ehu.es

Received 12 October 2014; Accepted 30 December 2014

Academic Editor: Calogero Vetro

Copyright (C) 2015 M. De la Sen and E. Karapınar. This is an open access article distributed under the Creative Commons Attribution License, which permits unrestricted use, distribution, and reproduction in any medium, provided the original work is properly cited.

\begin{abstract}
This paper investigates properties of convergence of distances of $p$-cyclic contractions on the union of the $p$ subsets of an abstract set $X$ defining probabilistic metric spaces and Menger probabilistic metric spaces as well as the characterization of Cauchy sequences which converge to the best proximity points. The existence and uniqueness of fixed points and best proximity points of $p$-cyclic contractions defined in induced complete Menger spaces are also discussed in the case when the associate complete metric space is a uniformly convex Banach space. On the other hand, the existence and the uniqueness of fixed points of the $p$-composite mappings restricted to each of the $p$ subsets in the cyclic disposal are also investigated and some illustrative examples are given.
\end{abstract}

\section{Introduction}

Fixed point theory in the framework of probabilistic metric spaces $[1-4]$ is receiving important research attention. See, for instance, [2-11]. On the other hand, Menger probabilistic metric spaces are a special case of the wide class of probabilistic metric spaces which are endowed with a triangular norm $[2,3,5,7,9,12,13]$. In probabilistic metric spaces, the deterministic notion of distance is considered to be probabilistic in the sense that, given any two points $x$ and $y$ of a metric space, a measure of the distance between them is a probabilistic metric $F_{x, y}(t)$, rather than the deterministic distance $d(x, y)$, which is interpreted as the probability of the distance between $x$ and $y$ being less than a real value $t(t>0)$, [3].

Fixed point theorems in complete Menger probabilistic metric spaces for probabilistic concepts of $B$ and $C$ contractions can be found in [2] together with a new notion of contraction, referred to as a $(\Psi, C)$-contraction. Such a contraction was proved to be useful for multivalued mappings while it generalizes the previous concept of $C$-contraction.
On the other hand, 2-cyclic $\phi$-contractions on intersecting subsets of complete Menger spaces were discussed in [5] for contractions based on control $\phi$-functions. See also [6]. It was found that fixed points are unique. Also, $\phi$-contractions in complete probabilistic Menger spaces have been also studied in [9] through the use of altering distances. On the other hand, probabilistic Banach spaces versus fixed point theory were discussed in [8]. The concept of probabilistic complete metric space was adapted to the formalism of Banach spaces defined with norms being defined by triangular functions and under a suitable ordering in the considered space. In parallel, mixed monotone operators in such Banach spaces were discussed while the existence of coupled minimal and maximal fixed points for these operators was analyzed and discussed in detail. Further extensions to contractive mappings in complete fuzzy metric spaces by using generalized distribution functions have been studied in $[6,7]$ and some references therein. The concept of altering distances was exploited in a very general context to derive fixed point results in [14], and extended later on in [12] to Menger probabilistic metric spaces. On the other hand, general fixed point 
theorems have been very recently obtained in [13] for two new classes of contractive mappings in Menger probabilistic metric spaces. The results have been established for $\alpha-\psi$ contractive mappings and for a generalized $\beta$-type one. It has also to be pointed out that the parallel background literature related to best proximity points and fixed points in cyclic mappings in metric and Banach spaces is exhaustive. See, for instance, [15-28] and references therein. Fixed point theory has also been widely applied to stability and equilibrium problems since, even based on intuition, the convergence of trajectory-solutions of differential or difference equations or dynamic systems to a point can be typically associated to the convergence of sequences to fixed points; see, for instance, $[27,29,30]$ and references therein, and to ergodic processes [31].

This paper investigates properties of convergence of distances of $p$-cyclic contractions on the union of the $p$ subsets of the abstract set $X$ defining the probabilistic metric spaces and the Menger probabilistic metric spaces as well as the characterization of Cauchy sequences which converge to best proximity points. The existence and the uniqueness of fixed points and best proximity points of $p$-cyclic contractions is also discussed in induced complete Menger probabilistic metric spaces in the case that the associate complete metric space is a uniformly convex Banach space. The fixed points of the $p$-composite mappings restricted to each of the $p$ subsets in the cyclic framework disposal are also investigated. Finally, some examples are discussed.

\section{Main Results}

Denote $\mathbf{R}_{+}=\mathbf{R}_{0+} \cup\{0\}=\{z \in \mathbf{R}: z>0\}, \mathbf{R}_{0+}=\{z \in \mathbf{R}$ : $z \geq 0\}, \mathbf{Z}_{+}=\mathbf{Z}_{0+} \cup\{0\}=\{z \in \mathbf{Z}: z>0\}, \mathbf{Z}_{0+}=\{z \in \mathbf{Z}: z \geq$ $0\}$, and $\bar{n}=\{1,2, \ldots, n\}$. Denote by $\mathbf{L}$ the set of distribution functions $H: \mathbf{R} \rightarrow[0,1]$, which are nondecreasing and left continuous, such that $F(0)=0$ and $\sup _{t \in \mathbf{R}} F(t)=1$. Let $X$ be a nonempty abstract set of elements and let $\mathbf{F}: X \times X \rightarrow \mathbf{L}$ be a mapping from $X \times X$ into the set of distribution functions L, which are symmetric functions of elements $F_{x, y}$ for every $(x, y) \in X \times X$. Then, the ordered pair $(X, F)$ is a probabilistic metric space (PM), [1-4], if

(1) $\forall x, y \in X\left(\left(F_{x, y}(t)=1 ; \forall t \in \mathbf{R}_{+}\right) \Leftrightarrow(x=y)\right)$,

(2) $F_{x, y}(t)=F_{y, x}(t) ; \forall x, y \in X, \forall t \in \mathbf{R}$,

(3) $\forall x, y, z \in X ; \forall t_{1}, t_{2} \in \mathbf{R}_{+},\left(\left(F_{x, y}\left(t_{1}\right)=F_{y, z}\left(t_{2}\right)=\right.\right.$ $\left.1) \Rightarrow\left(F_{x, z}\left(t_{1}+t_{2}\right)=1\right)\right)$.

Note that an interpretation of the PM-space is that $\mathbf{F}: X \times$ $X \rightarrow \mathbf{L}$ is a set of distribution functions. A particular distribution function $F_{x, y}$ is a probabilistic metric (or distance) which takes values $F_{x, y}(t)$, identified with the values of a mapping $H: \mathbf{R} \rightarrow[0,1]$ in the set of all the distribution functions $\mathbf{L}$, so that two points are (probabilistically) identical if the probabilistic metric in-between them gives probabilistic certainty (namely, probability equal to one) for a mutual distance being smaller than any given positive real number.
A Menger PM-space is a triplet $(X, F, \Delta)$, where $(X, F)$ is a PM-space which satisfies

$$
\begin{array}{r}
F_{x, y}\left(t_{1}+t_{2}\right) \geq \Delta\left(F_{x, z}\left(t_{1}\right), F_{z, y}\left(t_{2}\right)\right) \\
\forall x, y, z \in X, \quad \forall t_{1}, t_{2} \in \mathbf{R}_{0+}
\end{array}
$$

under $\Delta:[0,1] \times[0,1] \rightarrow[0,1]$ which is a $t$-norm (or triangular norm) belonging to the set $\mathbf{T}$ of $t$-norms which satisfy the properties

$$
\begin{aligned}
& \text { (1) } \Delta(a, 1)=a \\
& \text { (2) } \Delta(a, b)=\Delta(b, a) \\
& \text { (3) } \Delta(c, d) \geq \Delta(a, b) \text { if } c \geq a, d \geq b \\
& \text { (4) } \Delta(\Delta(a, b), c)=\Delta(a, \Delta(b, c)) .
\end{aligned}
$$

A property which is a consequence of the above ones is $\Delta(a, 0)=0$. The probabilistic diameter of a subset $A$ of $X$ is a function from $\mathbf{R}_{0+}$ to $[0,1]$ defined by $D_{A}^{p 0}(z)=\sup _{t<z} \inf _{x, y \in A} F_{x, y}(t)$ and $A$ which is probabilistically bounded if $D_{A}^{p}=\sup _{z \in \mathbf{R}_{+}} D_{A}^{p 0}(z)=1$ [2]. Note that the diameter of a set refers to the real interval length where the argument of the probabilistic metric is nonzero while the probabilistic diameter is a measure of boundedness or unboundedness of such a set. The (probabilistic) distance in-between the subsets $A$ and $B$ of $X$ defines the argument interval length of zero probability distance in-between points of two subsets $A$ and $B$ of $X$ and it is defined as

$$
\begin{aligned}
D & =d(A, B) \\
& =\inf \left(z \in \mathbf{R}_{0+}: \sup _{x \in A, y \in B} F_{x, y}(t)=0 ; \forall t \in[0, z]\right) .
\end{aligned}
$$

The concept of $p$-cyclic contraction in a PM-space is recalled below (see, e.g., [1-6]).

Definition 1. A mapping $T: \bigcup_{i \in \bar{p}} A_{i} \rightarrow \bigcup_{i \in \bar{p}} A_{i}$ is a $p$-cyclic contraction in a $(X, F) \mathrm{PM}$-space, where $A_{i}$ are nonempty subsets of $X$ with $D$ being the distance between the adjacent subsets $A_{i}$ and $A_{i+1}, \forall i \in \bar{p}$; if $T\left(A_{i}\right) \subseteq A_{i+1}, \forall i \in \bar{p}$ and there exists a real constant $K \in[0,1)$ such that, for each pair $(x, y) \in A_{i} \times A_{i+1}, \forall i \in \bar{p}$, the following constraint holds:

$$
F_{T x, T y}(K t+(1-K) D) \geq F_{x, y}(t) ; \quad \forall t(>D) \in \mathbf{R}_{+},
$$

equivalently, $F_{T x, T y}(t) \geq F_{x, y}\left(K^{-1}(t-D)+D\right) ; \forall t(>D) \in \mathbf{R}_{+}$, and

$$
F_{T x, T y}(t-D) \geq F_{x, y}\left(K^{-1}(t-D)\right) ; \quad \forall t(>D) \in \mathbf{R}_{+} .
$$

Note that, if $T: \bigcup_{i \in \bar{p}} A_{i} \rightarrow \bigcup_{i \in \bar{p}} A_{i}$ is $p$-cyclic contraction in a metric space $(X, d)$, then there is $K \in[0,1)$ such that, for each $(x, y) \in A_{i} \times A_{i+1}, \forall i \in \bar{p}$,

$$
d(T x, T y) \leq K d(x, y)+(1-K) D .
$$

The space $(X, F)$ is the induced PM-space of the metric space $(X, d)$ if $F_{x, y}(t)=H(t-d(x, y)) ; \forall x, y \in X ; \forall t \in \mathbf{R}_{+}$. 
The definitions of convergent sequence and Cauchy sequence in a PM-space follows.

Definition 2. A sequence $\left\{x_{n}\right\} \subset X$ converges to $x \in X$ if, for any given real constants $\varepsilon, \lambda \in \mathbf{R}_{+}$, with $\lambda \in(0,1)$, there is $N_{0}=N_{0}(\varepsilon, \lambda)$ such that $F_{x_{n}, x}(\varepsilon)>1-\lambda$ for $n \geq N_{0}$.

Definition 3. A sequence $\left\{x_{n}\right\}$ is a Cauchy sequence in $X$ if for any given real constants $\varepsilon, \lambda \in \mathbf{R}_{+}$, with $\lambda \in(0,1)$, there is $N_{0}=N_{0}(\varepsilon, \lambda)$ such that $F_{x_{n}, x_{m}}(\varepsilon)>1-\lambda$ for $n, m \geq N_{0}$.

Note that if $\left\{x_{n}\right\} \subset X$ converges to some $x \in X$ then $\lim _{n \rightarrow \infty} F_{x_{n}, x}(t)=1, \forall t \in \mathbf{R}_{+}$, and, if $\left\{x_{n}\right\}$ is a Cauchy sequence in $X$, then $\lim _{n, m \rightarrow \infty} F_{x_{n}, x_{m}}(t)=1, \forall t \in \mathbf{R}_{+}$. A Menger PM-space $(X, F, \Delta)$ is complete if every Cauchy sequence is convergent in $X$.

Definition 4. $x \in \mathrm{cl} A_{i}$ and $y \in \mathrm{cl} A_{i+1}$ are adjacent best proximity points so that the pair $(x, y) \in \operatorname{cl} A_{i} \times \operatorname{cl} A_{i+1}$ is an adjacent pair of best proximity points for any given $i \in \bar{p}$ if $F_{x, y}(t)=0 ; t \in(-\infty, D]$ and $F_{x, y}\left(D^{+}\right)=1$, where $D=d\left(A_{i}, A_{i+1}\right), \forall i \in \bar{p}$.

Since $F \quad: \quad \mathbf{R} \rightarrow[0,1]$ is nondecreasing and left continuous with $F(0)=F\left(0^{-}\right)=0$ then if $x, y$ are adjacent best proximity points $F_{x, y}(t)=F_{x, y}\left(D^{+}\right)=1$ for $t(>D) \in \mathbf{R}_{+}$. If $D=0$, that is, all subsets $A_{i} \subset X, \forall i \in \bar{p}$, pair-wise intersect, so that $\bigcap_{i \in \bar{p}} A_{i} \neq \varnothing$, then all the best proximity points are coincident at a unique fixed point in $\bigcap_{i \in \bar{p}} \mathrm{cl} A_{i}$ and $F_{x, T x}(t)=F_{x, y}\left(0^{+}\right)=1$ for $t \in \mathbf{R}_{+}$which implies $x=$ $T x=y$ from the first property of the probabilistic space. The subsequent result addresses the fact that the sequences built by iterations through $p$-cyclic contractions $T: \bigcup_{i \in \bar{p}} A_{i} \rightarrow$ $\bigcup_{i \in \bar{p}} A_{i}$ in complete Menger PM-spaces lead to convergent Cauchy sequences on each of the subsets $A_{i} \subset X, \forall i \in \bar{p}$, if such subsets are closed, the limits of the subsequences being in the closures of the subsets of the cyclic disposal in the general case. The convergence points of such subsequences are fixed points of the composite mappings $T^{p}$ restricted to each one of the subsets $A_{i}, \forall i \in \bar{p}$, and best proximity points located at each pair of the closures of the adjacent subsets $A_{i}$ and $A_{i+1}, \forall i \in \bar{p}$, and at the sets themselves if the subsets are closed.

Theorem 5. Assume that $T: \bigcup_{i \in \bar{p}} A_{i} \rightarrow \bigcup_{i \in \bar{p}} A_{i}$ is a $p$ cyclic contraction in a Menger PM-space $(X, F, \Delta)$ endowed with a $t$-norm $\Delta:[0,1] \times[0,1] \rightarrow[0,1]$, where $A_{i}$ are nonempty subsets of $X$ such that $D=d\left(A_{i}, A_{i+1}\right), \forall i \in \bar{p}$, under a probability density function $F: \mathbf{R} \rightarrow[0,1]$ such that $F_{x, y}(t)=0$ for $t \in(-\infty, D]$ and $F_{x, y}(t)=1, \forall t(>D) \in \mathbf{R}_{+}$, for any $(x, y) \in A_{i} \times A_{i+1}, \forall i \in \bar{p}$.

Then, the following property holds:

(i) $\lim _{t \rightarrow \infty} F_{T^{n p} x, T^{n p+1} x}(t)$

$$
\begin{gathered}
=\lim _{n \rightarrow \infty} \lim _{t \rightarrow D^{+}} F_{T^{n} x, T^{n+1} x}\left(K^{n} t+\left(1-K^{n}\right) D\right)=1 ; \\
\forall(x, y) \in A_{i} \times A_{i+1}, \quad \forall i \in \bar{p}, \quad \forall t>D,
\end{gathered}
$$

$$
\begin{aligned}
& \lim _{t \rightarrow D^{-}} F_{T^{n} x, T^{n} y}\left(K^{n} t+\left(1-K^{n}\right) D\right)=F_{T^{n} x, T^{n} y}(t)=0 ; \\
& \forall(x, y) \in A_{i} \times A_{i+1}, \quad \forall i \in \bar{p}, \quad \forall n \in \mathbf{Z}_{0+}, \quad t \in(-\infty, D] .
\end{aligned}
$$

Assume, furthermore, that the Menger PM-space $(X, F, \Delta)$ is complete and that $\Delta:[0,1] \times[0,1] \rightarrow[0,1]$ is continuous and $\Delta(t, t) \geq t$ for each $t \in \mathbf{R}_{+}$. Then, the subsequent additional property holds:

$$
\begin{array}{r}
\lim _{n \rightarrow \infty} F_{T^{n p} x, T^{(n+m) p_{x}}}(t)=\lim _{n \rightarrow \infty} F_{T^{n p} x, T^{(n+m) p} y}(t)=1 ; \\
\forall x \in \bigcup_{i \in \bar{p}} A_{i}, \quad \forall t(>D) \in \mathbf{R}_{+},
\end{array}
$$

with $x, y \in A_{j}$ for some $j \in \bar{p}$, and, for any given $\varepsilon \in \mathbf{R}_{+}$and $\lambda \in(0,1)$, there is $n_{0}=n_{0}(\varepsilon, \lambda)$ such that $\min \left(F_{T^{n p} x, T^{(n+m) p_{x}}}(t), F_{T^{n p} x, T^{n p} y}(t)\right)>1-\lambda, \forall n\left(\geq n_{0}\right) \in \mathbf{Z}_{0+}$, so that the sequences $\left\{T^{n p} x\right\} \subseteq A_{i},\left\{T^{n p+1} x\right\} \subseteq A_{i+1}$ are Cauchy sequences; then convergent to adjacent best proximity points, $x_{i}^{*} \in \operatorname{cl} A_{i}\left(x_{i}^{*} \in A_{i}\right.$ if $A_{i}$ is closed $)$ and $x_{i+1}^{*} \in \operatorname{cl} A_{i+1}\left(x_{i+1}^{*} \in\right.$ $A_{i+1}$ if $A_{i+1}$ is closed), $\forall x \in A_{i}, \forall i \in \bar{p}$, which are fixed points of the composite mapping $T^{p}$ on $A_{i}$ and $A_{i+1}$, respectively.

Proof. Note that $\left\{T^{n p} x\right\} \subset A_{i}$ and $\left\{T^{n p} y\right\} \subset A_{i+1}, \forall(x, y) \in$ $A_{i} \times A_{i+1}, \forall i \in \bar{p}$. Thus, one gets from (5) that

$$
\begin{aligned}
& F_{T^{n p} x, T^{n p} y}(t-D) \\
& \geq F_{T^{n p-1} x, T^{n p-1} y}\left(K^{-1}(t-D)\right) \\
& \geq \cdots \geq F_{x, y}\left(K^{-n p}(t-D)\right) ; \quad \forall t(>D) \in \mathbf{R}_{+} ;
\end{aligned}
$$

$\lim _{n \rightarrow \infty} F_{T^{n p} x, T^{n p} y}(t)=F_{x, y}(\infty)=1, \forall(x, y) \in A_{i} \times A_{i+1}$, $\forall t(>D) \in \mathbf{R}_{+}$. It turns out that we can fix the argument of the first left-hand side to $K t+(1-K) D$ and, correspondingly, its first right-hand side to $t$. In the same way, we can use for both arguments with the corresponding pairs $\left(t+\left(K^{-1}-1\right) D, K^{-1} t\right)$ and $\left(t, K^{-1}(t-D)+D\right)$. Furthermore, it is obvious that

$$
\begin{array}{r}
\lim _{n \rightarrow \infty} \lim _{t \rightarrow t_{0}^{-}} F_{T^{n p} x, T^{n p} y}(t)=F_{x, y}(D)=F\left(t_{0}\right)=0 ; \\
\forall(x, y) \in A_{i} \times A_{i+1}, \quad t_{0} \in(-\infty, D]
\end{array}
$$

and then, for any given $\varepsilon \in \mathbf{R}_{+}$and $\lambda \in(0,1)$, there is $N=$ $N(\varepsilon, \lambda) \in \mathbf{Z}_{0+}$ such that $F_{T^{n p} x, T^{n p} y}(D+\varepsilon)>1-\lambda$ for $n(>N) \epsilon$ $\mathbf{Z}_{0+}, \forall(x, y) \in A_{i} \times A_{i+1}, \forall t(>D) \in \mathbf{R}_{+}$. Hence, property (i) follows. On the other hand, note from property (i) for $y=T x$ and any $x \in \bigcup_{i \in \bar{p}} A_{i}$ that

$$
\lim _{t \rightarrow \infty} F_{T^{n p} x, T^{n p+1} x}(t)=1 ; \quad \forall t(>D) \in \mathbf{R}_{+}
$$

and that, for any given $\varepsilon \in \mathbf{R}_{+}$and $\lambda \in(0,1)$, there is $N_{1}=$ $N_{1}(\varepsilon, \lambda) \in \mathbf{Z}_{0+}$ such that $F_{T^{n p} x T^{n p+1} x}(D+\varepsilon)>1-\lambda$ for $n(>$ $\left.N_{1}\right) \in \mathbf{Z}_{0+}$. Then, one gets from (1), (5), the second and third 
conditions of (2), and the condition $\Delta(x, x) \geq x$ for each $x \in$ $[0,1]$ and $n, m \in \mathbf{Z}_{+}$:

$$
\begin{aligned}
& F_{T^{n p} x, T^{(n+m) p} x}(t) \\
& \geq \Delta\left(F_{T^{n p} x T^{n p+1} x}((1-K) t), F_{T^{n p+1} x, T^{(n+m) p_{x}}}(K t)\right) \\
& \geq \Delta\left(F_{x, T x}\left(\frac{1-K^{m p}}{1-K} K^{-n p}((1-K) t-D)+D\right),\right. \\
& \left.\Delta\left(F_{T^{n p+1} x, T^{n p+2} x}(K(1-K) t), F_{T^{n p+2} x, T^{(n+m) p_{x}}}\left(K^{2} t\right)\right)\right) \\
& \geq \Delta\left(F_{x, T x}\left(\frac{1-K^{m p}}{1-K} K^{-n p}((1-K) t-D)+D\right),\right. \\
& \Delta\left(F_{x, T x}\left(\frac{1-K^{m p}}{1-K} K^{-n p-1}(K(1-K) t-D)+D\right),\right. \\
& \left.\left.F_{T^{n p+2} x, T^{(n+m) p} x}\left(K^{2} t\right)\right)\right) \\
& =\Delta\left(F_{x, T x}\left(\frac{1-K^{m p}}{1-K} K^{-n p}((1-K) t-D)+D\right)\right. \text {, } \\
& \Delta\left(F_{x, T x}\left(\frac{1-K^{m p}}{1-K} K^{-n p}((1-K) t-D)+D\right),\right. \\
& \left.\left.F_{T^{n p+2} x, T^{(n+m) p_{x}}}\left(K^{2} t\right)\right)\right) \\
& \geq \Delta\left(F_{x, T x}\left(\frac{1-K^{m p}}{1-K} K^{-n p}((1-K) t-D)+D\right),\right. \\
& \Delta\left(F_{x, T x}\left(\frac{1-K^{m p}}{1-K} K^{-n p}((1-K) t-D)+D\right),\right. \\
& \left.\left.F_{T^{(n+m) p-1} x, T^{(n+m) p_{x}}}\left(K^{m p-1} t\right)\right)\right) \\
& \geq \Delta\left(F_{x, T x}\left(\frac{1-K^{m p}}{1-K} K^{-n p}((1-K) t-D)+D\right),\right. \\
& \Delta\left(F_{x, T x}\left(\frac{1-K^{m p}}{1-K} K^{-n p}((1-K) t-D)+D\right),\right. \\
& \left.\left.F_{x, T x}\left(K^{-(n+m) p+1}\left(K^{m p-1} t-D\right)+D\right)\right)\right) \\
& =\Delta\left(F_{x, T x}\left(\frac{1-K^{m p}}{1-K} K^{-n p}((1-K) t-D)+D\right)\right. \text {, } \\
& \Delta\left(F_{x, T x}\left(\frac{1-K^{m p}}{1-K} K^{-n p}((1-K) t-D)+D\right),\right. \\
& \left.\left.F_{x, T x}\left(\frac{1-K^{m p}}{1-K} K^{-n p}((1-K) t-D)+D\right)\right)\right) \\
& \geq \Delta\left(F_{x, T x}\left(\frac{1-K^{m p}}{1-K} K^{-n p}((1-K) t-D)+D\right),\right.
\end{aligned}
$$

$$
\begin{gathered}
\left.F_{x, T x}\left(\frac{1-K^{m p}}{1-K} K^{-n p}((1-K) t-D)+D\right)\right) \\
\geq F_{x, T x}\left(\frac{1-K^{m p}}{1-K} K^{-n p}((1-K) t-D)+D\right) ;
\end{gathered}
$$

$\forall t \in \mathbf{R}_{+}$.

Then, $\lim _{n \rightarrow \infty} F_{T^{n p} x, T^{(n+m) p_{x}}}(t)=F_{x, T x}(\infty)=1, \forall x \in \bigcup_{i \in \bar{p}} A_{i}$, $\forall t \in \mathbf{R}_{+}$since $F_{x, T x}(t)$ is nondecreasing with supremum over $t \in \mathbf{R}_{+}$equalizing unity. Then, for any given $\varepsilon \in \mathbf{R}_{+}$and $\lambda \epsilon$ $(0,1)$, there is $n_{0}=n_{0}(\varepsilon, \lambda)$ such that $F_{T^{n p} P_{X} T^{(n+m) p_{x}}}(t)>1-\lambda$, $\forall n\left(\geq n_{0}\right) \in \mathbf{Z}_{0+}$ so that $\left\{T^{n p} x\right\} \subset A_{j}$ is a Cauchy sequence which is convergent to a point in $\operatorname{cl}\left(A_{j}\right)$, since $(X, F, \Delta)$ is complete, if $x \in A_{j}$ for any $j \in \bar{p}$. Now, if we take $x, y(\neq x) \in$ $A_{i}$ for some arbitrary given $i \in \bar{p}$, one obtains in a similar way that

$$
F_{T^{n p} x, T^{n p} y}(t) \geq F_{x, y}\left(K^{-n p}((1-K) t-D)+D\right)
$$

and then $\lim _{n \rightarrow \infty} F_{T^{n p} x, T^{n p} y}(t)=1, \forall t \in \mathbf{R}_{+}$. Then, for any given $\varepsilon \in \mathbf{R}_{+}$and $\lambda \in(0,1)$, there is $n_{1}=n_{1}(\varepsilon, \lambda)$ such that $F_{T^{n p} x, T^{(n+m) p} y}(t)>1-\lambda, \forall n\left(\geq n_{01}\right) \in \mathbf{Z}_{0+}$ and so that $\left\{T^{n p} x\right\} \subset$ $A_{i}$ for any $x \in A_{i}$ is a Cauchy sequence, and a subsequence of $\left\{T^{n} x\right\} \subset \bigcup_{i \in \bar{p}} A_{i}$, which is convergent to a point in $\mathrm{cl}\left(A_{i}\right)$, since $(X, F, \Delta)$ is complete. Since $\left\{T^{n p} x\right\}$ and $\left\{T^{n p+1} x\right\}$ are convergent to $x_{i}^{*} \in \operatorname{cl}\left(A_{i}\right)$ and $x_{i+1}^{*} \in \operatorname{cl}\left(A_{i+1}\right)$ for any $x \in A_{i}$, any $i \in \bar{p}$, then one gets by taking into account property (i) that $x_{i}^{*}$ and $x_{i+1}^{*}$ are adjacent best proximity points; that is, $x_{i+1}^{*}=T x_{i}^{*}$. Assume the contrary, so that $\left\{T^{n p} x\right\} \rightarrow x_{i}^{*}$, $\left\{T^{n p+1} x\right\} \rightarrow x_{i+1}^{*}$, and $x_{i+1}^{*} \neq T x_{i}^{*}$ for some $x \in A_{i}$ and some $i \in \bar{p}$ so that:

$$
\begin{aligned}
& F_{x_{i}^{*}, x_{i+1}^{*}}(t) \\
& \geq \Delta\left(F_{T^{n p} x, x_{i}^{*}}((1-K) t), F_{T^{n p} x, x_{i+1}^{*}}(K t)\right) \\
& =\Delta\left(F_{T^{n p} x, x_{i}^{*}}((1-K) t),\right. \\
& \left.\quad \Delta\left(F_{T^{n p+1} x, T^{n p} x}\left(\frac{K t}{2}\right), F_{T^{n p+1} x, x_{i+1}^{*}}\left(\frac{K t}{2}\right)\right)\right) \\
& \geq \Delta\left(F_{T^{n p} x, x_{i}^{*}}\left(D^{+}\right), \Delta\left(F_{x, T x}\left(D^{+}\right), F_{T x, x_{i+1}^{*}}\left(D^{+}\right)\right)\right) ; \\
& \forall t(>D) \in \mathbf{R}_{+}
\end{aligned}
$$

and then $F_{x_{i}^{*}, x_{i+1}^{*}}(t)=1$ thus $x_{i+1}^{*}=T x_{i}^{*}, \forall x \in \bigcup_{i \in \bar{p}} A_{i}, \forall i \in \bar{p}$. It also turns out that those points are also fixed points of the restricted composite self-map $T^{p}$ to $A_{i}$ and, respectively, to $A_{i+1}$ since, otherwise, the respective sequences $\left\{T^{n p} x\right\}$ and $\left\{T^{n p+1} x\right\}$ would not be convergent. Property (ii) has been proved.

Note that Theorem 5 does not address the uniqueness of the best proximity points in the subsets $A_{i} \subset X, \forall i \in \bar{p}$. Their existence is guaranteed in the closures of the subsets $A_{i}$ since all sequences of the form $\left\{T^{n p} x\right\},\left\{T^{n p+1} x\right\}$ are Cauchy convergent sequences to two respective limits at distance 
$D$ with probability one allocated in adjacent sets $\mathrm{cl} A_{i}$ and $\operatorname{cl} A_{i+1}$ for any given initial point $x \in A_{i}$ for any $i \in \bar{p}$. The subsequent result addresses the existence and the uniqueness of best proximity points if $(X,\|\|)$ is a uniformly convex Banach space and $(X, F, \Delta)$ is a complete Menger PM-space provided that the subsets $A_{i} \subset X, \forall i \in \bar{p}$, are closed and convex.

The following Corollary to Theorem 5 follows for the case when $A_{i}=A \subset X, \forall i \in \bar{p}$ by making $D=0$.

Corollary 6. Assume that $T: A \rightarrow A$ is a contraction in a Menger PM-space $(X, F, \Delta)$ endowed with a t-norm $\Delta:[0,1] \times$ $[0,1] \rightarrow[0,1]$, where $A$ is a nonempty subset of $X$ under a probability density function $F: \mathbf{R} \rightarrow[0,1]$ such that $F_{x, y}(t)=$ 0 for $t \in(-\infty, 0]$ and $F_{x, y}(t)=1$ for $t>0$ for any given $x, y \in A$.

Then, the following property holds:

$$
\begin{aligned}
& \text { (i) } \lim _{t \rightarrow \infty} F_{T^{n} x, T^{n+1} x}(t)=\lim _{n \rightarrow \infty} F_{T^{n} x, T^{n+1} x}\left(0^{+}\right)=1 \text {, } \\
& \forall x, y \in A, \quad \forall i \in \bar{p}, \quad \forall t>0, \\
& \lim _{t \rightarrow 0^{-}} F_{T^{n} x, T^{n} y}\left(K^{n} t\right)=F_{T^{n} x, T^{n} y}(t)=0, \\
& \forall(x, y) \in A_{i} \times A_{i+1}, \quad \forall i \in \bar{p}, \quad \forall n \in \mathbf{Z}_{0+}, \\
& t \in(-\infty, 0] \text {. }
\end{aligned}
$$

If, furthermore, the Menger PM-space $(X, F, \Delta)$ is complete and that $\Delta:[0,1] \times[0,1] \rightarrow[0,1]$ is continuous and $\Delta(t, t) \geq t$ for each $t \in \mathbf{R}_{+}$. Then, the subsequent additional property holds:

(ii) $\lim _{n \rightarrow \infty} F_{T^{n} x, T^{n+m} x}(t)=\lim _{n \rightarrow \infty} F_{T^{n} x, T^{(n+m)} y}(t)=1$,

$$
\forall x, y \in A, \quad \forall t \in \mathbf{R}_{+},
$$

with $x, y \in A$, and, for any given $\varepsilon \in \mathbf{R}_{+}$and $\lambda \in(0,1)$, there is $n_{0}=n_{0}(\varepsilon, \lambda) \in \mathbf{Z}_{0+}$ such that $\min \left(F_{T^{n} x, T^{n+m} x}(t), F_{T^{n} x, T^{n} y}(t)\right)>$ $1-\lambda, \forall n\left(\geq n_{0}\right) \in \mathbf{Z}_{0+}$, so that the sequence $\left\{T^{n} x\right\} \subseteq A$ is a Cauchy sequence, then convergent to a fixed point $x^{*} \epsilon$ $\operatorname{cl} A$ ( $x^{*} \in A$ if $A$ is closed $)$ which is also a fixed point of the composite mapping $T^{p}$ on $A$.

A second main result of the paper and a corresponding corollary for the case when all the subsets of the cyclic disposal coincide follow below.

Theorem 7. Assume that $T: \bigcup_{i \in \bar{p}} A_{i} \rightarrow \bigcup_{i \in \bar{p}} A_{i}$ is a $p$-cyclic contraction in a PM-space $(X, F)$, where $A_{i}$ are nonempty subsets of $X$ such that $D=d\left(A_{i}, A_{i+1}\right), \forall i \in \bar{p}$, endowed with a probability density function $F: \mathbf{R} \rightarrow[0,1]$ such that $F_{x, y}(t)=0$ for $t \in(-\infty, D]$ and $F_{x, y}(t)=1$ for $t>D$ for any $(x, y) \in A_{i} \times A_{i+1}, \forall i \in \bar{p}$. Then, the following properties hold:

$$
\begin{aligned}
& \text { (i) } \lim _{n \rightarrow \infty} \lim _{t \rightarrow D^{+}} F_{T^{n} x, T^{n} y}\left(K^{n} t+\left(1-K^{n}\right) D\right)=1 \text {; } \\
& \forall(x, y) \in A_{i} \times A_{i+1}, \quad \forall i \in \bar{p}, \quad \forall t>D,
\end{aligned}
$$

$$
\begin{array}{r}
\lim _{t \rightarrow D^{-}} F_{T^{n} x, T^{n} y}\left(K^{n} t+\left(1-K^{n}\right) D\right)=F_{T^{n} x, T^{n} y}(t)=0 ; \\
\forall(x, y) \in A_{i} \times A_{i+1}, \quad \forall i \in \bar{p}, \quad \forall n \in \mathbf{Z}_{0+}, \\
t \in(-\infty, D] .
\end{array}
$$

Assume, furthermore, that $(X, d) \equiv(X,\|\|)$ is a uniformly convex Banach space under a complete norm-induced metric $d: X \times X \rightarrow \mathbf{R}_{0+}$, so that $(X, F, \Delta)$ is a complete Menger PMspace under the distribution function $F_{x, y}(t)=H(t-d(x, y))$, $\forall x, y \in X$, for any $t \in \mathbf{R}$ subject to

$$
\begin{array}{r}
F_{x, y}(t)=0 \quad \text { if } t \leq D, \\
F_{x, y}(t)=1 \quad \text { if } t>D ; \\
\forall(x, y) \in A_{i} \times A_{i+1}, \quad \forall i \in \bar{p} .
\end{array}
$$

Assume also that the subsets $A_{i}$ of $X$ are closed and convex, $\forall i \in \bar{p}$. Then, the following two further properties hold.

(ii) Consider sequences $\left\{T^{n} x\right\},\left\{T^{n} z\right\},\left\{T^{n} y\right\} \subset \bigcup_{i \in \bar{p}} A_{i}$ for any $x, z \in A_{i}$ and $y \in A_{i+1}$ for some arbitrarily given $i \in \bar{p}$ such that

(1) $F_{T^{n p} x, T^{n p} z}\left(D^{+}\right) \rightarrow 1$ as $n \rightarrow \infty$,

(2) $F_{T^{m p} x, T^{n p} y}(D+\varepsilon)>1-\lambda$ for all $m>n \geq N_{1}$ and each given $\varepsilon, \lambda(<1) \in \mathbf{R}_{+}$and some $N_{1}=$ $N_{1}(\varepsilon, \lambda)$.

Then, for each given $\varepsilon, \lambda(<1) \in \mathbf{R}_{+}$, there exists $N \in \mathbf{Z}_{0+}$ such that $F_{T^{m p} x, T^{n p} z}(\varepsilon)>1-\lambda$ for all $m>n \geq N$; then $\lim _{n, m(>n) \rightarrow \infty} \lim _{\varepsilon \rightarrow 0^{+}} F_{T^{m p} x, T^{n p} z}(\varepsilon)=1$.

(iii) $\left\{T^{n p} x\right\} \subset A_{i},\left\{T^{n p} z\right\} \subset A_{i}$, and $\left\{T^{n p} y\right\} \subset A_{i+1}$ are Cauchy sequences for any $x, z \in A_{i}, y \in A_{i+1}$, and any given $i \in \bar{p}$ and then bounded, and convergent to unique fixed points $y_{i}=T^{p} y_{i}$ and $x_{i}=z_{i}=T^{p} z_{i}$ of the composite mapping $T^{p}$ restricted to each $A_{i}$ into itself, $\forall i \in \bar{p}$. Also, $z_{i}$ and $T z_{i}$ are the unique best proximity points in the adjacent subsets $A_{i}$ and $A_{i+1}$ of $X, \forall i \in \bar{p}$.

Proof. Since $T: \bigcup_{i \in \bar{p}} A_{i} \rightarrow \bigcup_{i \in \bar{p}} A_{i}$ is a $p$-cyclic contraction in a PM-space $(X, F)$, one gets from (4) for any $(x, y) \in A_{i} \times$ $A_{i+1}, \forall i \in \bar{p}$, that

$$
\begin{aligned}
& F_{T^{n} x, T^{n} y}\left(K^{n} t+\left(1-K^{n}\right) D\right) \\
& \geq \cdots \geq F_{T^{2} x, T^{2} y}\left(K^{2} t+K(1-K) D+(1-K) D\right) \\
& =F_{T^{2} x, T^{2} y}\left(K^{2} t+\left(1-K^{2}\right) D\right) \\
& \geq F_{T x, T y}(K t+(1-K) D) \geq F_{x, y}(t) ; \quad t \in \mathbf{R} .
\end{aligned}
$$


Thus, since $F: \mathbf{R} \rightarrow[0,1]$ is nondecreasing and leftcontinuous,

$$
\begin{aligned}
& \lim _{n \rightarrow \infty} \lim _{t \rightarrow D^{+}} F_{T^{n} x, T^{n} y}\left(K^{n} t+\left(1-K^{n}\right) D\right) \\
& \quad=\lim _{n \rightarrow \infty} F_{T^{n} x, T^{n} y}\left(D^{+}\right)=1 ; \\
& \forall(x, y) \in A_{i} \times A_{i+1}, \quad \forall i \in \bar{p}, \quad \forall t>D \\
& \lim _{n \rightarrow \infty} \lim _{t \rightarrow D^{-}} F_{T^{n} x, T^{n} y}\left(K^{n} t+\left(1-K^{n}\right) D\right) \\
& \quad=\lim _{n \rightarrow \infty} F_{T^{n} x, T^{n} y}\left(D^{+}\right)=F_{T^{n} x, T^{n} y}(t)=0 ; \\
& \forall(x, y) \in A_{i} \times A_{i+1}, \quad \forall i \in \bar{p}, \quad \forall n \in \mathbf{Z}_{0+}, \quad t \in(-\infty, D] .
\end{aligned}
$$

Hence, property (i) has been proved. To prove property (ii), first note that $F_{x, y}(t)=H(t-d(x, y))=0, \forall(x, y) \in A_{i} \times A_{i+1}$, for any $t(\leq D) \in \mathbf{R}$ since $(t-d(x, y)) \leq 0$ and then $F_{x, y}(t)=$ $H(0)=0$ and also $F_{x, y}(t)=H(t-d(x, y))=0$ for $t>D$.

We now proceed by contradiction. Assume the contrary. Then, $\exists \varepsilon_{0}, \lambda_{0}(\leq 1) \in \mathbf{R}_{+}$such that, for each $k \in \mathbf{Z}_{0+}$, there

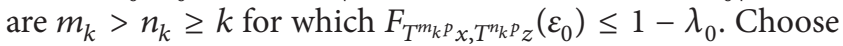
$0<\gamma<1$ such that $\varepsilon_{0} / \gamma>D$ and choose $0<\varepsilon<\min \left(\left(\varepsilon_{0} / \gamma\right)-\right.$ $D, D \delta(\gamma) /(1-\delta(\gamma)))$. For such an $\varepsilon \in \mathbf{R}_{+}$, there is $\lambda(<1) \in \mathbf{R}_{+}$ and $N_{1}, N_{2} \in \mathbf{Z}_{+}$such that, since $F_{T^{n} k^{p} z, T^{n} k^{p} y}(t+\varepsilon) \rightarrow 1$ as $n \rightarrow \infty, \forall t \geq D$, then $F_{T^{n_{k} p} z, T^{n} p^{p}}(t+\varepsilon)>1-\lambda$ for $m_{k}>$ $n_{k} \geq N_{2}$. Since, furthermore, $F_{T^{m} p^{p} x, T^{n_{k}} p_{y}}(t+\varepsilon)>1-\lambda$ for $m_{k}>n_{k} \geq N_{1}, \forall t>D$, and some $N_{1}, N_{2} \in \mathbf{Z}_{0+} \operatorname{since}(X, F, \Delta)$ is a Menger PM-space. Then, if $N=\max \left(N_{1}, N_{2}\right)$, since $A_{i}$ are closed and convex, $\forall i \in \bar{p}$, and $(X,\|\|)$ is a uniformly convex (and hence reflexive) Banach space with modulus of convexity $\delta(\varepsilon)>0$ for $\varepsilon>0$ and $\delta(\cdot)$ is strictly increasing, one has that, for any $x, y, p \in X, R \in \mathbf{R}_{+}$and $r \in[0,2 R]$,

$$
\begin{gathered}
{[(\|x-p\| \leq R) \wedge(\|y-p\| \leq R) \wedge\|x-y\| \geq r]} \\
\Longrightarrow\left\|\frac{x+y}{2}-p\right\| \leq\left(1-\delta\left(\frac{r}{R}\right)\right) R
\end{gathered}
$$

and then, for any $x, z \in A_{i}, y \in A_{i+1}$ and any arbitrary given $i \in \bar{p}$,

$$
\begin{aligned}
1 & >1-\lambda_{0} \geq F_{\left(T^{m_{k} p_{x}} T^{n_{k} p} z\right) / 2, T^{n_{k}} p_{y}}(t+\varepsilon) \\
& =H\left(t+\varepsilon-\left\|\frac{T^{m_{k} p} x+T^{n_{k} p} z}{2}-T^{n_{k} p} y\right\|\right) \\
& \geq H\left(t+\varepsilon-\left(1-\delta\left(\frac{\varepsilon_{0}}{D+\varepsilon}\right)\right)(D+\varepsilon)\right) ; \quad t>D
\end{aligned}
$$

and there is $t_{1}>D$ such that the following contradiction is got if $\left\{T^{n p} z\right\}$ is not a Cauchy sequence in the Menger PM-space $(X, F, \Delta)$ so that $F_{T^{m}{ }_{k} p_{x}, T^{n}{ }^{n} p_{z}}\left(\varepsilon_{0}\right) \leq 1-\lambda_{0}$ for $m_{k}>n_{k} \geq k$ and some $\varepsilon_{0}, \lambda_{0}(\leq 1) \in \mathbf{R}_{+}$:

$$
1 \geq H\left(t+\varepsilon-\left(1-\delta\left(\frac{\varepsilon_{0}}{D+\varepsilon}\right)\right)(D+\varepsilon)\right)=1 ; \quad t \geq t_{1} .
$$

Hence, property (ii) follows. To prove property (iii), note from property (ii) that, for any $i \in \bar{p}$ and initial points $x=z \in A_{i}$, one has that $F_{T^{m p} z, T^{n p} z}(\varepsilon)>1-\lambda$ for $m>$ $N \geq N_{0}$ and some $N_{0}=N_{0}(\varepsilon, \lambda)$ and any $\varepsilon, \lambda(<1) \in \mathbf{R}_{+}$, then $\lim _{m(>n) n \rightarrow \infty} F_{T^{m p} z, T^{n p} z}\left(0^{+}\right)=1$ so that $\left\{T^{n p} x\right\} \subset A_{i}$ is a Cauchy sequence for any $x \in A_{i}$ and any $i \in \bar{p}$ and then bounded, which is convergent to some $z_{i} \in A_{i}$ since the Menger PM-space $(X, F, \Delta)$ is complete. For the other two Cauchy sequences, the same proof applies. Since the sequence $\left\{T^{n p} x\right\} \subset A_{i}$ is convergent to $z_{i} \in A_{i}$ (since $A_{i}$ is closed) then $\lim _{n \rightarrow \infty} F_{T^{n p} z, z_{i}}\left(0^{+}\right)=1$. Assume that $z_{i} \neq T^{p} z_{i}$ for some $i \in \bar{p}$. Then, since $\left\{T^{(n+1) p} z\right\} \rightarrow z_{i}$ does not converge to $T^{p} z_{i} \neq z_{i}$ then $1>\lim _{n \rightarrow \infty} F_{T^{(n+1) p} z, T^{p} z_{i}}\left(0^{+}\right)=$ $\lim _{n \rightarrow \infty} F_{T^{p}\left(T^{n p} z\right), T^{p} z_{i}}\left(0^{+}\right)=1$ so that $\left\{T^{(n+1) p} z\right\} \rightarrow T^{p} z_{i} \neq z_{i}$ for such an $i \in \bar{p}$, a contradiction. Thus, $z_{i}=T^{p} z_{i}, \forall i \in \bar{p}$. It is now proved by contradiction that $z_{i}=T^{p} z_{i}$ is unique, $\forall i \in \bar{p}$. Assume that $z_{i}=T^{p} z_{i} \neq \bar{z}_{i}=T^{p} \bar{z}_{i}$ for some $i \in \bar{p}$. Then,

$$
\begin{aligned}
1 & =F_{T^{n p} z_{i}, T^{n p} \bar{z}_{i}}(t)=\lim _{n \rightarrow \infty} F_{T^{n}\left(T^{p} z_{i}\right), T^{n}\left(T^{p} z_{i}\right)}(t) \\
& =F_{z_{i}} \bar{z}_{i}(t) ; \quad \forall t \in \mathbf{R}_{+} ; \forall i \in \bar{p} \\
& \Longleftrightarrow z_{i}=\bar{z}_{i} ; \quad \forall i \in \bar{p}
\end{aligned}
$$

which contradicts $z_{i} \neq \bar{z}_{i}$ for some $i \in \bar{p}$. Thus, the fixed points of the restricted composite mapping $T^{p}$ to each $A_{i}$ are all unique, $\forall i \in \bar{p}$. Now, assume that $z_{i+1}=T z_{i}=T^{p+1} z_{i}$, $\forall i \in \bar{p}$, is false; that is, $z_{i+1} \neq T z_{i}$ for $z_{i+1}$ and $T z_{i}$ is some $A_{i+1}$ for some $i \in \bar{p}$. Then, since $T: \bigcup_{i \in \bar{p}} A_{i} \rightarrow \bigcup_{i \in \bar{p}} A_{i}$ is a $p$-cyclic contraction in $(X, F, \Delta)$, one has the following contradiction for some $t_{1} \in \mathbf{R}_{+}$, positive reals:

$$
\begin{aligned}
1 & >F_{T z_{i}, z_{i+1}}\left(t_{1}\right)=F_{T^{n p+1} z_{i}, T^{n p} z_{i+1}}\left(t_{1}\right) \\
& \geq \lim _{n \rightarrow \infty} \lim _{x \rightarrow D^{+}} F_{T^{n p}\left(T z_{i}\right), T^{n p} z_{i+1}}\left(K^{-n p}(t-D)+x\right) \\
& =F_{z_{i+1}, T z_{i}}(t)=F_{z_{i+1}, T z_{i}}\left(D^{+}\right)=1 ; \quad \forall t(>D) \in \mathbf{R}_{+} .
\end{aligned}
$$

Then, $z_{i+1} \neq T z_{i}, \forall i \in \bar{p}$.

Corollary 8. Assume that $T: A \rightarrow A$ is a contraction in a PM-space $(X, F)$, where $A \subset X$ is nonempty subsets of $X$, endowed with a probability density function $F: \mathbf{R} \rightarrow[0,1]$ such that $F_{x, y}(t)=0$ for $t \in(-\infty, 0]$ and $F_{x, y}(t)=1$ for $t>0$ for any $x, y \in A$. Then, the following properties hold:

$$
\begin{aligned}
& \text { (i) } \lim _{n \rightarrow \infty} \lim _{t \rightarrow 0^{+}} F_{T^{n} x, T^{n} y}\left(K^{n} t\right)=1, \quad \forall x, y \in A, \forall t>0 \text {, } \\
& \lim _{t \rightarrow 0^{-}} F_{T^{n} x, T^{n} y}\left(K^{n} t\right)=F_{T^{n} x, T^{n} y}(t)=0,
\end{aligned}
$$$$
\forall x, y \in A, \quad \forall n \in \mathbf{Z}_{0+}, \quad t \in(-\infty, 0] .
$$

Assume, furthermore, that $(X, d) \equiv(X,\|\|)$ is a uniformly convex Banach space under a complete norm-induced metric 
$d: X \times X \rightarrow \mathbf{R}_{0+}$, so that $(X, F, \Delta)$ is a complete Menger PMspace under the distribution function $F_{x, y}(t)=H(t-d(x, y))$, $\forall x, y \in X$ for any $t \in \mathbf{R}$ subject to

$$
\begin{array}{r}
F_{x, y}(t)=0 \quad \text { if } t \leq 0, \\
F_{x, y}(t)=1 \quad \text { if } t>0, \\
\forall x, y \in A .
\end{array}
$$

Assume also that $A$ is closed and convex. Then consider the following.

(ii) Consider sequences $\left\{T^{n} x\right\},\left\{T^{n} z\right\},\left\{T^{n} y\right\} \subset A$ for any $x, y, z \in A$ such that

(1) $F_{T^{n} x, T^{n} z}\left(0^{+}\right) \rightarrow 1$ as $n \rightarrow \infty$;

(2) $F_{T^{m} x, T^{n} y}(\varepsilon)>1-\lambda$ for all $m>n \geq N_{1}$ and each given $\varepsilon, \lambda(<1) \in \mathbf{R}_{+}$and some $N_{1}=N_{1}(\varepsilon, \lambda)$.

Then, for each given $\varepsilon, \lambda(<1) \in \mathbf{R}_{+}$, there exists $N \in \mathbf{Z}_{0+}$ such that $F_{T^{m} x, T^{n} z}(\varepsilon)>1-\lambda$ for all $m>n \geq N$ and $\lim _{n, m(>n) \rightarrow \infty} \lim _{\varepsilon \rightarrow 0^{+}} F_{T^{m} x, T^{n} z}(\varepsilon)=1$.

(iii) $\left\{T^{n} x\right\}$ is a Cauchy sequence for any $x \in A$ and then bounded, and convergent to a unique fixed point $z=$ $T z$.

Remark 9. Note that if $T: \bigcup_{i \in \bar{p}} A_{i} \rightarrow \bigcup_{i \in \bar{p}} A_{i}$ is a $p$-cyclic contraction in $(X, F)$ then the composite mappings $T^{p}$ from $\bigcup_{i \in \bar{p}} A_{i} \mid A_{j}$ into $A_{j}, \forall j \in \bar{p}$, satisfy from (4) for each $x, y \in$ $A_{j} ; \forall j \in \bar{p}$ that:

$$
F_{T^{p} x, T^{p} y}(\bar{K} t+(1-\bar{K}) D) \geq F_{x, y}(t) ; \quad \forall t \in \mathbf{R}
$$

with $\bar{K}=K^{p} \in[0,1)$. However, each composite mapping is not, in general, a contraction on each $A_{i}, \forall i \in p$, although any iterated sequences $\left\{T^{n p} x\right\} \subset A_{i}$ are Cauchy sequences, $\forall i \in p$, in a complete Menger PM-space $(X, F, \Delta)$.

The following result is a direct consequence of Theorem 7 if the subsets of the cyclic disposal intersect.

Corollary 10. Assume that all the hypothesis of Theorem 7 hold while $\bigcap_{i \in \bar{p}} A_{i} \neq \varnothing$; that is, the sets $A_{i}, \forall i \in \bar{p}$, are not disjoint. Then, the following properties hold:

(i) $\quad T: \bigcup_{i \in \bar{p}} A_{i} \longrightarrow \bigcup_{i \in \bar{p}} A_{i}, \quad T^{p}: \bigcup_{i \in \bar{p}} A_{i} \mid A_{j} \longrightarrow A_{j}$;

$\forall j \in \bar{p}$ are contractions in $(X, F)$,

$$
\begin{array}{r}
\lim _{n \rightarrow \infty} \lim _{t \rightarrow 0^{+}} F_{T^{n} x, T^{n} y}\left(K^{n} t\right)=1 ; \\
\forall(x, y) \in A_{i} \times A_{i+1}, \quad \forall i \in \bar{p}, \quad \forall t>0, \\
\operatorname{Lim}_{t \rightarrow 0^{-}} F_{T^{n} x, T^{n} y}\left(K^{n} t\right)=F_{T^{n} x, T^{n} y}(t)=0 ; \\
\forall(x, y) \in A_{i} \times A_{i+1}, \quad \forall i \in \bar{p}, \quad \forall n \in \mathbf{Z}_{0+}, \\
t \in(-\infty, 0] .
\end{array}
$$

Assume, furthermore, that $(X, d) \equiv(X,\|\|)$ is a uniformly convex Banach space under a norm-induced metric $d: X \times$ $X \rightarrow \mathbf{R}_{0+}$, so that $(X, F, \Delta)$ is a complete Menger PM-space under the distribution function $F_{x, y}(t)=H(t-d(x, y))$, $\forall x, y \in X$, for any $t \in \mathbf{R}$ subject to

$$
\begin{aligned}
F_{x, y}(t)=0 & \text { if } t \leq 0, \\
F_{x, y}(t)=1 & \text { if } t>0 ; \\
\forall(x, y) \in A_{i} \times A_{i+1}, & \forall i \in \bar{p} .
\end{aligned}
$$

Assume also that the subsets $A_{i}$ of $X$ are closed and convex, $\forall i \in \bar{p}$. Then, the following two further properties hold.

(ii) Consider the sequences $\left\{T^{n} x\right\},\left\{T^{n} z\right\},\left\{T^{n} y\right\} \subset \bigcup_{i \in \bar{p}} A_{i}$ for any $x, z \in A_{i}$ and $y \in A_{i+1}$ for some arbitrarily given $i \in \bar{p}$ such that:

(1) $F_{T^{n p} x, T^{n p} z}\left(0^{+}\right) \rightarrow 1$ as $n \rightarrow \infty$,

(2) $F_{T^{m p} x, T^{n p} y}(\varepsilon)>1-\lambda$ for all $m>n \geq N_{1}$ and each given $\varepsilon, \lambda(<1) \in \mathbf{R}_{+}$and some $N_{1}=N_{1}(\varepsilon, \lambda)$.

Then, for each given $\varepsilon, \lambda(<1) \in \mathbf{R}_{+}$, there exists $N \in \mathbf{Z}_{0+}$ such that $F_{T^{m p} x, T^{n p} z}(\varepsilon)>1-\lambda$ for all $m>n \geq N$; then $\lim _{n, m(>n) \rightarrow \infty} \lim _{\mathcal{E} \rightarrow 0^{+}} F_{T^{m p} x, T^{n p} \mathcal{Z}}(\varepsilon)=1$.

(iii) $\left\{T^{n p} x\right\} \subset A_{i},\left\{T^{n p} z\right\} \subset A_{i}$, and $\left\{T^{n p} y\right\} \subset A_{i+1}$ are Cauchy sequences for any $x, z \in A_{i}, y \in A_{i+1}$, and any given $i \in \bar{p}$, then bounded, and convergent to a unique fixed point $z \in \bigcap_{i \in \bar{p}} A_{i}$ of $T: \bigcup_{i \in \bar{p}} A_{i} \rightarrow \bigcup_{i \in \bar{p}} A_{i}$ and $T^{p}: \bigcup_{i \in \bar{p}} A_{i} \mid A_{j} \rightarrow A_{j}, \forall j \in \bar{p}$.

Proof. The proof of Theorem 7(i) and the fact that the sequences of Theorem 7(iii) are Cauchy sequences are particular cases of Theorem 11 and do not need a proof. Now, assume that two adjacent best proximity points are distinct, namely, $z_{i+1} \neq T z_{i}$ for some $i \in \bar{p}$. Then, in the same way as in the last part of the proof of Theorem 7, one gets the contradiction

$$
\begin{aligned}
1 & >F_{T z_{i}, z_{i+1}}(t) \geq \lim _{n \rightarrow \infty} \lim _{x \rightarrow D^{+}} F_{T^{n p}\left(T z_{i}\right), T^{n p} z_{i+1}}\left(K^{-n p} t+x\right) \\
& =F_{z_{i+1}, T z_{i}}(t)=F_{z_{i+1}, T z_{i}}\left(0^{+}\right)=1 ; \quad \forall t \in \mathbf{R}_{+},
\end{aligned}
$$

together with the parallel contradiction to $T z_{i} \neq z_{i}$ for some $i \in \bar{p}$ :

$$
\begin{aligned}
1 & >F_{T z_{i}, z_{i}}(t) \geq \lim _{n \rightarrow \infty} \lim _{x \rightarrow D^{+}} F_{T^{n p}\left(T z_{i}\right), T^{n p} z_{i}}\left(K^{-n p} t+x\right) \\
& =F_{z_{i}, T z_{i}}(t)=F_{z_{i}, T z_{i}}\left(0^{+}\right)=1 ; \quad \forall t \in \mathbf{R}_{+} .
\end{aligned}
$$

Thus, $z_{i+1}=z_{i}=T z_{i}, \forall i \in \bar{p}$, which is in $\bigcap_{i \in \bar{p}} A_{i}$, since $\bigcap_{i \in \bar{p}} A_{i}$ is nonempty and closed.

The subsequent result formulates a connection between metric spaces and their induced Menger PM-spaces [3] concerning some basic properties of $p$-cyclic contractions whose contractive condition is defined on the metric space $(X, d)$ rather than on the Menger PM-space. 
Theorem 11. Let $(X, d)$ be a metric space, let $(X, F)$ be an induced PM-space, where $\mathbf{F}: X \times X \rightarrow \mathbf{L}, H: R \rightarrow[0,1]$ in $\mathbf{L}$ being defined by $F_{x, y}(t)=H(t-d(x, y)), \forall x, y \in X, \forall t \in \mathbf{R}$. Let $T: \bigcup_{i \in \bar{p}} A_{i} \rightarrow \bigcup_{i \in \bar{p}} A_{i}$ be a p-cyclic contraction in $(X, d)$; that is,

$$
\begin{array}{r}
d(T x, T y) \leq K d(x, y)+(1-K) D ; \\
\forall(x, y) \in A_{i} \cup A_{i+1} ; \quad \forall i \in \bar{p}
\end{array}
$$

for some real constant $K \in[0,1)$, where $A_{i}$ are nonempty subsets of $X, \forall i \in \bar{p}$, such that $D=d\left(A_{i}, A_{i+1}\right), \forall i \in \bar{p}$.

Then, the following properties hold:

$$
\begin{gathered}
\text { (i) } \lim _{n \rightarrow \infty} \lim _{t \rightarrow D^{+}} F_{T^{n} x, T^{n} y}(t)=H\left(D^{+}\right)=1 ; \\
\forall(x, y) \in A_{i} \cup A_{i+1}, \quad \forall i \in \bar{p}, \\
\lim _{n \rightarrow \infty} \lim _{t \rightarrow D^{-}} F_{T^{n} x, T^{n} y}(t)=H\left(D^{-}\right)=0 ; \\
\forall(x, y) \in A_{i} \cup A_{i+1}, \quad \forall i \in \bar{p}, \\
F_{x, y}(t)=0 ; \quad \forall(x, y) \in A_{i} \cup A_{i+1}, \quad \forall i \in \bar{p} ; \forall t \in(-\infty, D] .
\end{gathered}
$$

(ii) Assume, in addition, that $(X, d) \equiv(X,\|\|)$ is a uniformly convex Banach space under a norm-induced metric, so that $(X, F, \Delta)$ is an induced complete probabilistic Menger PM-space under the $\Delta$-norm of the minimum. Assume also that the subsets $A_{i}$ of $X$ are closed and convex, $\forall i \in \bar{p}$. Then, $\left\{T^{n p} x\right\} \subset A_{i}$ is a Cauchy sequence and then bounded and convergent to a unique fixed point $z_{i}=T^{p} z_{i}$ of the composite mapping $T^{p}$ restricted to $A_{i}$ into itself, $\forall i \in \bar{p}$, while $z_{i}$ and $T z_{i}$ are the unique best proximity points in the adjacent subsets $A_{i}$ and $A_{i+1}$ of $X, \forall i \in \bar{p}$. There is a unique fixed point $z=T z$ which is coincident with all the $p$ best proximity points if the subsets $A_{i}, \forall i \in \bar{p}$, have a nonempty intersection.

Proof. If $\mathbf{F}: X \times X \rightarrow F$ is the mapping induced by the metric $d: X \times X \rightarrow \mathbf{R}_{0+}$ and $T: \bigcup_{i \in \bar{p}} A_{i} \rightarrow \bigcup_{i \in \bar{p}} A_{i}$ is a $p$-cyclic contraction then there is a real constant $K \in[0,1)$ such that

$$
\begin{array}{r}
d(T x, T y) \leq K d(x, y)+(1-K) D ; \\
\forall(x, y) \in A_{i} \cup A_{i+1} ; \quad \forall i \in \bar{p}
\end{array}
$$

and $(X, F)$ is an induced PM-space by the metric space $(X, d)$. Thus, one gets from (35) in accordance with the definition $F_{x, y}(t)=H(t-d(x, y)), \forall(x, y) \in A_{i} \cup A_{i+1}, \forall i \in \bar{p}$, for any $t \in \mathbf{R}$, and since $H: \mathbf{R} \rightarrow \mathbf{R}_{0+}$ is nondecreasing and left-continuous and $d(x, y) \geq D$ that

$$
\begin{aligned}
& F_{T x, T y}(K t+(1-K) D) \\
& \quad=H(K t+(1-K) D-d(T x, T y))
\end{aligned}
$$

$$
\begin{aligned}
& \geq H(K(t-d(x, y))) \geq H(K(t-D)) \\
& =F_{x, y}(K(t-D)+d(x, y)) \\
& \geq F_{x, y}(K(t-D)+D) \geq F_{x, y}(D),
\end{aligned}
$$

$\forall(x, y) \in A_{i} \cup A_{i+1}, \forall i \in \bar{p}, \forall t(>D) \in \mathbf{R}_{+}$. Then, by proceeding recursively with (36) since $T\left(A_{i}\right) \subseteq A_{i+1}, \forall i \in \bar{p}$, one gets

$$
\begin{aligned}
& F_{T^{n} x, T^{n} y}(K t+(1-K) D) \geq F_{x, y}(D) ; \\
& \forall(x, y) \in A_{i} \cup A_{i+1}, \quad \forall i \in \bar{p}, \quad \forall n \in \mathbf{Z}_{0+}, \quad \forall t(>D) \in \mathbf{R}_{+} \\
& \lim _{n \rightarrow \infty} \inf _{t \rightarrow D^{+}}\left[F_{T^{n} x, T^{n} y}\left(K^{n} t+\left(1-K^{n}\right) t\right)-F_{x, y}(t)\right] \geq 0,
\end{aligned}
$$

$\forall(x, y) \in A_{i} \cup A_{i+1}, \forall i \in \bar{p}, \forall t(>D) \in \mathbf{R}_{+}$. Then, there exists the $\operatorname{limit}_{\lim _{n \rightarrow \infty}} \lim _{t \rightarrow D^{+}} F_{T^{n} x, T^{n} y}(t)=H\left(D^{+}\right)=1, \forall(x, y) \epsilon$ $A_{i} \cup A_{i+1}, \forall i \in \bar{p}$. In the same way, and since $F_{x, y}\left(D^{-}\right)=$ $H\left(D^{-}\right)=0 ; \forall(x, y) \in A_{i} \cup A_{i+1}$, one gets

$$
\begin{gathered}
\lim _{n \rightarrow \infty} \inf _{t \rightarrow D^{-}}\left[F_{T^{n} x, T^{n} y}\left(K^{n} t+\left(1-K^{n}\right) t\right)-F_{x, y}(t)\right] \\
=H\left(D^{-}\right) ; \quad \forall(x, y) \in A_{i} \cup A_{i+1}, \quad \forall i \in \bar{p} .
\end{gathered}
$$

Note from (1) that if $t \leq D$ then

$$
\begin{array}{r}
F_{T x, T y}(K t+(1-K) D) \leq H(D-d(T x, T y))=H(0)=0 ; \\
\forall(x, y) \in A_{i} \cup A_{i+1}, \quad \forall i \in \bar{p}
\end{array}
$$

so that $H(t)=H\left(D^{-}\right)=H(0)=0$ for $t \in[0, D]$ and property (i) has been proved. Property (ii) follows if $(X, F, \Delta)$ is now a Menger PM-space under the triangular norm $\Delta$ of the minimum induced by the metric space $(X, d)$. Note that $T: \bigcup_{i \in \bar{p}} A_{i} \rightarrow \bigcup_{i \in \bar{p}} A_{i}$ is a $p$-cyclic contraction, $(X,\|\|) \equiv(X, d)$ is a uniformly convex Banach space, and then a complete metric space and the subsets $A_{i}$ of $X$ are closed and convex, $\forall i \in \bar{p}$. Thus, from Theorem 7 (see also [4] for the deterministic framework), $(X, F, \Delta)$ is a complete Menger PM-space under the $\Delta t$-norm of the minimum defined by $\Delta(a, b)=\min (a, b)$. Then, for any given $\varepsilon \in \mathbf{R}_{+}$ and $\lambda \in(0,1)$ there is $N=N(\varepsilon, \lambda), F_{T^{n p} x, T^{n p+1}{ }_{x}}(D+\varepsilon) \geq$ $1-\lambda, F_{T^{n p} x, T^{(n+m) p_{x}}}(\varepsilon) \geq 1-\lambda$, and $F_{T^{n p} x, z_{i}}(\varepsilon) \geq 1-\lambda$, $\forall n(>N) \in \mathbf{Z}_{0+}$, some unique $z_{i} \in A_{i}$, any given $x \in A_{i}$, and $i \in \bar{p}$. Thus, $\left\{T^{n p} x\right\} \subset A_{i}$ is a Cauchy sequence, then bounded, and convergent to a unique fixed point $z_{i}=T^{p} z_{i}$ of the composite mapping $T^{p}$ restricted to $A_{i}$ into itself, $\forall i \in \bar{p}$, while $z_{i}$ and $T z_{i}$ are the unique best proximity points in the adjacent subsets $A_{i}$ and $A_{i+1}$ of $X, \forall i \in \bar{p}$. 


\section{Examples}

Example 1. Assume that the PM-space $(X, F)$ is induced by a metric space $(X, d)$ and that $T: \bigcup_{i \in \bar{p}} A_{i} \rightarrow \bigcup_{i \in \bar{p}} A_{i}$ is a $p$-cyclic contraction in $(X, d)$ with contractive constant $K \in$ $(0,1)$, then in $(X, F)$ with $D$ being the distance in-between adjacent subsets $A_{i}$ and $A_{i+1}, \forall i \in \bar{p}$. If $x \in \bigcup_{j \in \bar{p}} A_{j}$ then for any $t(>d(x, T x)) \in \mathbf{R}_{+}$and any given $\varepsilon \in \mathbf{R}_{+}$and any given $\lambda \in(0,1)$,

$$
\begin{aligned}
& F_{T^{n} x, T^{n+1} x}(D+\varepsilon) \\
& \quad=F_{T^{n} x, T^{n+1} x}\left(K^{n} t+\left(1-K^{n}\right) D\right) \\
& \quad=H\left(K^{n} t+\left(1-K^{n}\right) D-d\left(T^{n} x, T^{n+1} x\right)\right) \\
& \quad \geq H\left(K^{n}(t-d(x, T x))\right)=1>1-\lambda
\end{aligned}
$$

for all integer $n \geq n_{0}$ and some integer $n_{0}=n_{0}(\varepsilon, \lambda)$. Since $d\left(T^{n} x, T^{n+1} x\right) \leq K^{n}(d(x, T x)-D)+D$, the condition $t>$ $d(x, T x)$ implies that $H\left(K^{n}(t-d(x, T x))\right) \geq H\left(0^{+}\right)=1$ and then $K^{n} t+\left(1-K^{n}\right) D \leq D+\varepsilon$ for $n \geq n_{0} \geq \ln ((t-D) / \varepsilon) /|\ln K|$. Assume that $x \in A_{i}$ for some $i \in \bar{p}$. If the probability of the distance in-between $x \in A_{i}$ and Tx $\in A_{i+1}$ is less than $t$ for some real $t>d(x, y)$ then the probability of the distance between $T^{n+\ell} x \in A_{i+j}$ and $T^{n+\ell+1} x \in A_{i+j+1}$ being less than $(D+\varepsilon)$ in the PM-space $(X, F)$, for some $j \in \overline{p-1} \cup\{0\}$ and any $\ell \in \mathbf{Z}_{0+}$, holds for any integer $n \geq \ln ((t-D) / \varepsilon) /|\ln K|$. Such an integer $j \in \overline{p-1} \cup\{0\}$ satisfies uniquely the constraints $n=m p+i+j \leq(m+1) p+i-1$ for the given integers $i \in \bar{p}, n \in \mathbf{Z}_{0+}$; that is, $j \equiv n-i(\bmod p)$. The (deterministic) distances $d\left(T^{n+\ell} x, T^{n+\ell+1} x\right) \leq D+\varepsilon$ in the metric space $(X, d)$ for any integer $n \geq n_{0} \geq \ln ((d(x, T x)-D) / \varepsilon) /|\ln K|$ provided that $d(x, T x)>D$. For any $x \in A_{i}$ and some arbitrary given $i \in \bar{p}$, the subsequences $\left\{T^{n p} x\right\} \subset A_{i}$ and $\left\{T^{n p+1} x\right\} \subset A_{i+1}$ of $\left\{T^{n} x\right\},\left\{T^{n+1} x\right\} \subset \bigcup_{j \in \bar{p}} A_{j}$, which are Cauchy convergent sequences, that is $\left\{T^{n p} x\right\} \rightarrow z_{i}\left(\in A_{i}\right)$ and $\left\{T^{n p+1} x\right\} \rightarrow z_{i+1}\left(\in A_{i+1}\right)$, satisfy the same constraints if $n \geq n_{0} \geq(1 / p|\ln K|) \ln ((t-D) / \varepsilon)$, respectively, $n \geq$ $n_{0} \geq(1 / p|\ln K|) \ln ((t-D) / \varepsilon)$ for any $t>d(x, T x) \geq D$ (see Theorem 5). The points $z_{i}$ and $z_{i+1}$ are adjacent to best proximity points of $T$ (being identical and a unique fixed point of $T$ if the subsets $A_{i}$ intersect) and fixed points of the composite map $T^{p}$ restricted to $A_{i}$ and of $T^{p}$ restricted to $A_{i+1}$, respectively.

Also, if $(X, F, \Delta)$ is a complete Menger PM-space, then the subsequences $\left\{T^{n p} x\right\} \subset A_{i}$, and $\left\{T^{n p+1} x\right\} \subset A_{i+1}$ are Cauchy and convergent sequences. Note that

$$
\begin{aligned}
& d\left(T^{n p} x, T^{(n+1) p} x\right) \\
& \leq \sum_{j=0}^{p-1} d\left(T^{n p+j} x, T^{n p+j-1} x\right) \\
& \leq \sum_{j=0}^{p-1} K^{j}\left(d\left(T^{n p+j} x, T^{n p+j+1} x\right)-D\right)+p D
\end{aligned}
$$

$$
\begin{aligned}
& \leq \sum_{j=0}^{p-1} K^{j}\left(d\left(T^{n p} x, T^{n p+1} x\right)-D\right)+p D \\
& =\frac{1-K^{p}}{1-K}\left(d\left(T^{n p} x, T^{n p+1} x\right)-D\right)+p D \\
& \leq \frac{1-K^{p}}{1-K}\left(K^{n p}(d(x, T x)-D)+D\right)+p D \\
& \leq \frac{1-K^{p}}{1-K} K^{n p} d(x, T x)+\left[\frac{1-K^{p}}{1-K}\left(1-K^{n p}\right)+p\right] D
\end{aligned}
$$

for all integer $n \geq n_{01}$ and some integer $n_{01}=n_{01}(\varepsilon, \lambda)$, for any given $\varepsilon \in \mathbf{R}_{+}$and $\lambda \in(0,1)$. Then,

$$
\begin{aligned}
& F_{T^{n p} x, T^{(n+1) p} x}(D+\varepsilon) \\
& =H\left(\frac{1-K^{p}}{1-K}\left(K^{n p}(t-D)+D\right)+p D-d\left(T^{n} x, T^{n+1} x\right)\right) \\
& =H\left(\frac{1-K^{p}}{1-K} K^{n p}(t-D)\right)=1>1-\lambda,
\end{aligned}
$$

such that $D+\varepsilon \geq\left(\left(1-K^{p}\right) /(1-K)\right)\left(K^{n p}(t-D)+D\right)+p D$ which holds for $n \geq n_{01}$ with

$$
\begin{gathered}
n_{01} \geq \frac{1}{|\ln K|} \ln \frac{1-K^{p}}{(1-K) \varepsilon} K^{n p}(t-D) \\
+\frac{D}{\varepsilon}\left(p+\frac{K\left(1-K^{p-1}\right)}{1-K}\right) .
\end{gathered}
$$

This can be refined with Theorem 7(ii) (see (12)) leading to the particular case for $m=1$ :

$$
\begin{array}{r}
F_{T^{n p} x, T^{(n+1) p} x}(t) \geq F_{x, T x}\left(K^{-n p}((1-K) t-D)+D\right) ; \\
\forall t(>D) \in \mathbf{R}_{+}
\end{array}
$$

implying $\lim _{n \rightarrow \infty} F_{T^{n p} x, T^{(n+1) p_{x}}}(\varepsilon)=\lim _{t \rightarrow \infty} F_{x, T x}(t)=1$ and $F_{T^{n p} x, T^{(n+1) p} x}(t)>1-\lambda, \forall t \in \mathbf{R}_{+}$for any given arbitrary $\varepsilon \in \mathbf{R}_{+}$, $\lambda \in(0,1)$, all integer $n \geq n_{02}$ and a sufficiently large integer number $n_{02}=n_{02}(\varepsilon)$. This implies that the subsequences $\left\{T^{n p} x\right\} \subset A_{i}$, and $\left\{T^{n p+1} x\right\} \subset A_{i+1}$ are Cauchy and convergent and contained in $(\varepsilon, \lambda)$-neighborhoods $U_{p_{j}}(\varepsilon, \lambda)=\left\{q_{j} \in X\right.$ : $\left.F_{p_{j}, q_{j}}(\varepsilon)>1-\lambda\right\}$ for $n \geq n_{02}$ which are centred at some $p_{j}=p_{j}(x) \in \operatorname{cl} A_{j}$ for $j=i, i+1$.

Example 2. Assume that the PM- space $(X, F)$ is induced by a metric space $(X, d)$ and that $\left\{T_{n}\right\}$ are possible sequences of $p$-cyclic mappings with $T_{n}: \bigcup_{i \in \bar{p}} A_{i} \rightarrow \bigcup_{i \in \bar{p}} A_{i}$ in $(X, d)$ with an associate sequence of constants $\left\{K_{n}\right\} \in(0, \infty)$ with $D$ being the distance in-between adjacent subsets $A_{i}$ and $A_{i+1}$, $\forall i \in \bar{p}$.

Furthermore, assume that $K_{n} \in K_{c}=K_{c s} \cup K_{c u}$ where $K_{c s}=\left\{L_{1}, L_{2}, \ldots, L_{n_{s}}\right\}$ and $K_{c u}=\left\{L_{n_{s}+1}, L_{n_{s}+2}, \ldots, L_{n_{s}+n_{u}}\right\}$ are disjoint and $K_{c s}$ is nonempty. It is assumed that $L_{i} \in$ $\left(0, \gamma_{0}\right) \subset(0,1)$ for $i \in \bar{n}_{s}$, with at least one $K \in K_{c u}$ satisfying 
$K \geq p^{-1}$ for some prefixed real constant $p \in(0,1)$ and $L_{i} \in\left[1, \gamma_{1}\right)$ for $i \in\left[n_{s+1}, n_{s}+n_{u}\right]$. Note that there is at least one member of $K_{c}$ which is also a member of the set $K_{c s}$ of value in $(0, p) \subset(0,1)$.

Now, construct sequences $\left\{x_{n}\right\}$ defined by $x_{n}=T x_{n-1}$ for $x_{0} \in \bigcup_{i \in \bar{p}} A_{i}$ and all $n \in \mathbf{Z}_{+}$subject to

$$
\begin{array}{r}
F_{x_{n+2}, x_{n+1}}\left(K_{n+1} t+\left(1-K_{n+1}\right) D\right) \geq F_{x_{n+1}, x_{n}}(t), \\
\forall t(>D) \in \mathbf{R}_{+}, \quad \forall n \in \mathbf{Z}_{0+},
\end{array}
$$

equivalently,

$$
\begin{array}{r}
F_{x_{n+2}, x_{n+1}}(t) \geq F_{x_{n+1}, x_{n}}\left(K_{n+1}^{-1}(t-D)+D\right), \\
\forall t(>D) \in \mathbf{R}_{+}, \quad \forall n \in \mathbf{Z}_{0+} .
\end{array}
$$

Assume that the objective is to construct admissible sequences of cyclic self-mappings of the given class so as to keep a distance $D_{0} \in[D, D+p \gamma \varepsilon]$ for some prefixed real constant $\gamma>1$ with guaranteed probability in-between consecutive sequence points of value at least $1-\lambda$ for some given real constants $\lambda, \varepsilon \in(0,1)$. It follows from the theoretical framework that for composite $p$-cyclic mappings $\widehat{T}_{n}: \bigcup_{i \in \bar{p}} A_{i} \rightarrow \bigcup_{i \in \bar{p}} A_{i}, \forall n \in \mathbf{Z}_{+}$of the form $\widehat{T}_{n}=T_{n} \circ \widehat{T}_{n-1}$ for $n \in \mathbf{Z}_{+}$with contractive constants in the set $K_{c s}=\left\{L_{1}, L_{2}, \ldots, L_{n_{s}}\right\}$, that is, of values less than one, one has that $\lim _{n \rightarrow \infty} F_{x_{n+2}, x_{n+1}}(t)=\lim _{t \rightarrow \infty} F_{x_{1}, x_{0}}(t)=1$, $\forall t(>D) \in \mathbf{R}_{+}$. Thus, there is $n_{0}=n_{0}(\varepsilon, \lambda) \in \mathbf{Z}_{0+}$ such that $F_{x_{n+2}, x_{n+1}}(t) \geq F_{x_{1}, x_{0}}(D+\varepsilon)>1-\lambda, \forall t(>D+\varepsilon) \in \mathbf{R}_{+}$, $\forall n\left(\geq n_{0}\right)$.

Then, a composite mapping $\widehat{T}_{n}=T_{n} \circ \widehat{T}_{n-1}$ for $n \in \mathbf{Z}_{+}$ is built with any sequence $\left\{T_{n}\right\}$ of $p$-cyclic mappings $T_{n}$ : $\bigcup_{i \in \bar{p}} A_{i} \rightarrow \bigcup_{i \in \bar{p}} A_{i}$ that satisfies the property $F_{x_{n+2}, x_{n+1}}(t)>$ $1-\lambda, \forall t(>D) \in \mathbf{R}_{+}, \forall n\left(\geq n_{0}\right)$, if the following steps are followed for its construction.

(a) $T_{n}$ are taken with contractive constants in the set $K_{c s}$ for $n \in \bar{n}_{0} \cup\{0\}$ and some $n_{0} \in \mathbf{Z}_{0+}$. Then, $F_{x_{n_{0}+1}, x_{n_{0}}}(t)>$ $1-\lambda, \forall t(>D+\varepsilon) \in \mathbf{R}_{+}$. It suffices that $F_{x_{n_{0}+1}, x_{n_{0}}}(D+\varepsilon)>$ $1-\lambda$ since the distribution function is nondecreasing.

(b) $T_{n}$ are taken with constants in the set $K_{c}$ as being contractive, expansive or nonexpansive depending on the values of their respective constants in the set $K_{c}$ for any integer $n>n_{0}$ such that the inequalities below hold:

$$
\begin{aligned}
& F_{x_{n+2}, x_{n+1}}(t) \geq F_{x_{n+1}, x_{n}}(D+\rho) \\
& \geq F_{x_{n_{0}+1}, x_{n_{0}}}(D+\rho) \\
& \geq F_{x_{1}, x_{0}}(D+\varepsilon)>1-\lambda ; \\
& \forall t(>D+\rho) \in \mathbf{R}_{+}
\end{aligned}
$$

for any $\rho \in[\varepsilon, \gamma \varepsilon)$. If the above chain of inequalities fails for $t=D+\gamma \varepsilon$, some tentative self-mapping $T_{n+1}^{\prime}$ and some $n_{1}(>$ $\left.n_{0}+1\right) \in \mathbf{Z}_{0+}$, then $T_{n+1}^{\prime}$ is changed to $T_{n+1}$ with contractive constant $K_{n+1}$ so that to guarantee

$$
\begin{aligned}
F_{x_{n+2}, x_{n+1}}(t) \geq & F_{x_{n+1}, x_{n}}(D+p \gamma \varepsilon) \\
\geq & F_{x_{n_{0}+1}, x_{n_{0}}}(D+\gamma \mathcal{\varepsilon}) \\
\geq & F_{x_{1}, x_{0}}(D+\varepsilon)>1-\lambda ; \\
& \forall t(>D+p \gamma \varepsilon) \in \mathbf{R}_{+}
\end{aligned}
$$

provided from (46) that $K_{n+1}^{-1}(t-D)+D \leq D+p \gamma \varepsilon$ for $t=$ $D+\gamma \varepsilon$; that is, $T_{n+1}$ is chosen to satisfy $1>K_{n+1} \geq p^{-1}$, $\forall n \in \mathbf{Z}_{0+}$.

(c) For $n \in\left(n_{1}+1, n_{2}\right]$, repeat Step (a) for some $n_{2}>n_{1}+1$ such that $F_{x_{n_{2}+1}, x_{n_{2}}}(D+\varepsilon)>1-\lambda$.

Example 3. Let $(X, F, \Delta)$ be a Menger PM-space, where $X=$ $\{\alpha, \beta, \gamma, \delta\}$ with subsets $A=\{\alpha, \beta, \delta\}$ and $B=\{\gamma, \delta\}, \Delta$ is the minimum $t$-norm and define the 2 -cyclic mapping $T: A \cup$ $B \rightarrow A \cup B$ as follows: $T \alpha=\gamma, T \beta=T \delta=T \gamma=\delta$, and let $F$ be defined by

$$
\begin{gathered}
F_{\beta, \delta}(t)=F_{\beta, \gamma}(t)= \begin{cases}0, & \text { if } t \leq 0, \\
1, & \text { if } t>0,\end{cases} \\
F_{\gamma, \alpha}(t)=F_{\delta, \gamma}(t)=F_{\delta, \alpha}(t)= \begin{cases}0, & \text { if } t \leq 0, \\
0.5, & \text { if } 0<t<4, \\
1, & \text { if } t \geq 3 .\end{cases}
\end{gathered}
$$

Note that $A \cap B=\{\delta\}$ so that these two sets intersect; then $D=d(A, B)=0, T: A \cup B \rightarrow A \cup B$ is a 2-cyclic contraction for any real constant $K \in(0,1)$, since $F_{\delta, \delta}(t)=1, \forall t \in \mathbf{R}_{+}$, and

$$
\begin{aligned}
& F_{T^{2} \alpha, T^{2} \gamma}(t) \geq F_{T \gamma, T \delta}\left(K^{-1} t\right) \geq F_{\delta, \delta}\left(K^{-2} t\right), \\
& F_{T^{2} \alpha, T^{2} \delta}(t) \geq F_{T \gamma, T \delta}\left(K^{-1} t\right) \geq F_{\delta, \delta}\left(K^{-2} t\right), \\
& F_{T^{2} \beta, T^{2} \gamma}(t) \geq F_{T \delta, T \delta}\left(K^{-1} t\right)=F_{\delta, \delta}\left(K^{-2} t\right), \\
& F_{T^{2} \beta, T^{2} \delta}(t) \geq F_{T \delta, T \delta}\left(K^{-1} t\right)=F_{\delta, \delta}\left(K^{-2} t\right), \\
& F_{T^{2} \delta, T^{2} \gamma}(t)=F_{T \delta, T \delta}\left(K^{-1} t\right)=F_{\delta, \delta}\left(K^{-2} t\right)
\end{aligned}
$$

for any real $\forall t \in \mathbf{R}_{+}$. Thus, $(X, F, \Delta)$ is a complete Menger PM-space and $\delta \in A \cap B$ is the unique fixed point of $T$ : $A \cup B \rightarrow A \cup B$ to which any sequences generated through $T$ converge in accordance with Theorem 5 . A close example has been discussed in [6] related to a cyclic contraction in a 2-Menger PM-space.

\section{Conflict of Interests}

The authors declare that they have no competing interests.

\section{Acknowledgments}

The authors are grateful to the Spanish Government for its support of this research with Grant DPI2012-30651 and to the 
Basque Government for its support of this research through Grants IT378-10 and SAIOTEK S-PE12UN015. They are also grateful to the University of Basque Country for its financial support through Grant UFI 2011/07. Finally, the authors thank the referees for their useful comments.

\section{References}

[1] B. Schweizer and A. Sklar, Probabilistic Metric Spaces, NorthHolland, Amsterdam, The Netherlands, 1983.

[2] E. Pap, O. Hadzic, and R. Mesiar, "A fixed point theorem in probabilistic metric spaces and an application," Journal of Mathematical Analysis and Applications, vol. 202, no. 2, pp. 433449, 1996.

[3] V. M. Sehgal and A. T. Bharucha-Reid, "Fixed points of contraction mappings on probabilistic metric spaces," Theory of Computing Systems, vol. 6, no. 1, pp. 97-102, 1972.

[4] B. Schweizer and A. Sklar, "Statistical metric spaces," Pacific Journal of Mathematics, vol. 10, pp. 313-334, 1960.

[5] B. S. Choudhury, K. Das, and S. K. Bhandari, "Fixed point theorem for mappings with cyclic contraction in Menger spaces," International Journal of Pure and Applied Sciences and Technology, vol. 4, no. 1, pp. 1-9, 2011.

[6] B. S. Choudhury, K. Das, and S. K. Bhandari, "Cyclic contraction result in 2-Menger space," Bulletin of International Mathematical Virtual Institute, vol. 2, no. 1, pp. 223-234, 2012.

[7] I. Beg and M. Abbas, "Fixed point and best approximation in Menger convex metric spaces," Archivum Mathematicum (BRNO). Tomus, vol. 41, pp. 389-397, 2005.

[8] I. Beg, A. Latif, R. Ali, and A. Azam, "Coupled fixed points of mixed monotone operators on probabilistic Banach spaces," Archivum Mathematicum, vol. 37, no. 1, pp. 1-8, 2001.

[9] D. Miheț, "Altering distances in probabilistic Menger spaces," Nonlinear Analysis: Theory, Methods \& Applications, vol. 71, no. 7-8, pp. 2734-2738, 2009.

[10] D. Mihet, "A Banach contraction theorem in fuzzy metric spaces," Fuzzy Sets and Systems, vol. 144, no. 3, pp. 431-439, 2004.

[11] S. Sedghi, B. S. Choudhury, and N. Shobe, "Strong common coupled fixed point result in fuzzy metric spaces," Journal of Physical Science, vol. 17, pp. 1-9, 2013.

[12] B. S. Choudhury and K. P. Das, "A new contraction principle in Menger spaces," Acta Mathematica Sinica, vol. 24, no. 8, pp. 1379-1386, 2008.

[13] D. Gopal, M. Abbas, and C. Vetro, "Some new fixed point theorems in Menger PM-spaces with application to Volterra type integral equation," Applied Mathematics and Computation, vol. 232, pp. 955-967, 2014.

[14] M. S. Khan, M. Swaleh, and S. Sessa, "Fixed point theorems by altering distances between the points," Bulletin of the Australian Mathematical Society, vol. 30, no. 1, pp. 1-9, 1984.

[15] A. A. Eldred and P. Veeramani, "Existence and convergence of best proximity points," Journal of Mathematical Analysis and Applications, vol. 323, no. 2, pp. 1001-1006, 2006.

[16] M. de la Sen, "Linking contractive self-mappings and cyclic Meir-Keeler contractions with Kannan self-mappings," Fixed Point Theory and Applications, vol. 2010, Article ID 572057, 2010.

[17] M. de la Sen, R. P. Agarwal, and N. Nistal, "Non-expansive and potentially expansive properties of two modified p-cyclic self-maps in metric spaces," Journal of Nonlinear and Convex Analysis, vol. 14, no. 4, pp. 661-686, 2013.
[18] M. De la Sen and R. P. Agarwal, "Fixed point-type results for a class of extended cyclic self-mappings under three general weak contractive conditions of rational type," Fixed Point Theory and Applications, vol. 2011, article 102, 2011.

[19] M. de la Sen, "On best proximity point theorems and fixed point theorems for $p$-cyclic hybrid self-mappings in Banach spaces," Abstract and Applied Analysis, vol. 2013, Article ID 183174, 14 pages, 2013.

[20] S. Karpagam and S. Agrawal, "Best proximity point theorems for $p$-cyclic Meir-Keeler contractions," Fixed Point Theory and Applications, vol. 2009, Article ID 197308, 2009.

[21] T. Suzuki, "Some notes on Meir-Keeler contractions and Lfunctions," Bulletin of the Kyushu Institute of Technology, no. 53, pp. 12-13, 2006.

[22] C. Di Bari, T. Suzuki, and C. Vetro, "Best proximity points for cyclic Meir-Keeler contractions," Nonlinear Analysis, Theory, Methods and Applications, vol. 69, no. 11, pp. 3790-3794, 2008.

[23] M. Derafshpour, S. Rezapour, and N. Shahzad, "On the existence of best proximity points of cyclic contractions," Advances in Dynamical Systems and Applications, vol. 6, no. 1, pp. 33-40, 2011.

[24] S. Rezapour, M. Derafshpour, and N. Shahzad, "Best proximity points of cyclic $\varphi$-contractions on reflexive Banach spaces," Fixed Point Theory and Applications, vol. 2010, Article ID 946178, 7 pages, 2010.

[25] M. A. Al-Thagafi and N. Shahzad, "Convergence and existence results for best proximity points," Nonlinear Analysis: Theory, Methods and Applications, vol. 70, no. 10, pp. 3665-3671, 2009.

[26] W. Sanhan, C. Mongkolkeha, and P. Kumam, "Generalized proximal $\psi$-contraction mappings and best proximity points," Abstract and Applied Analysis, vol. 2012, Article ID 896912, 19 pages, 2012.

[27] M. de la Sen and E. Karapinar, "Best proximity points of generalized semicyclic impulsive self-mappings: applications to impulsive differential and difference equations," Abstract and Applied Analysis, vol. 2013, Article ID 505487, 16 pages, 2013.

[28] E. Karapinar and I. M. Erhan, "Cyclic contractions and fixed point theorems," Filomat, vol. 26, no. 4, pp. 777-782, 2012.

[29] X. L. Qin, S. Y. Cho, and L. Wang, "Algorithms for treating equilibrium and fixed point problems," Fixed Point Theory and Applications, vol. 2013, article 308, 2013.

[30] K. S. Kim, J. K. Kim, and W. H. Lim, "Convergence theorems for common solutions of various problems with nonlinear mapping," Journal of Inequalities and Applications, vol. 2014, article 2, 2014.

[31] J. K. Kim, G. Li, Y. M. Nam, and K. . Kim, "Nonlinear ergodic theorems for almost-orbits of asymptotically nonexpansive type mappings in Banach spaces," Bulletin of the Korean Mathematical Society, vol. 38, no. 3, pp. 587-603, 2001. 


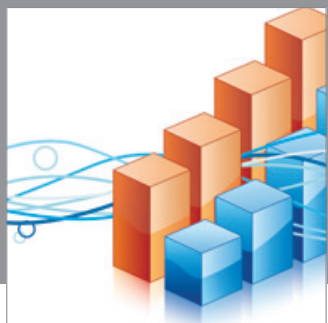

Advances in

Operations Research

mansans

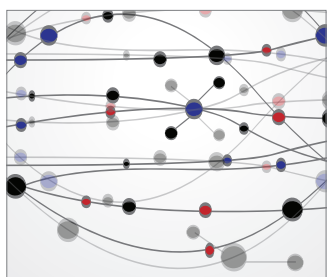

The Scientific World Journal
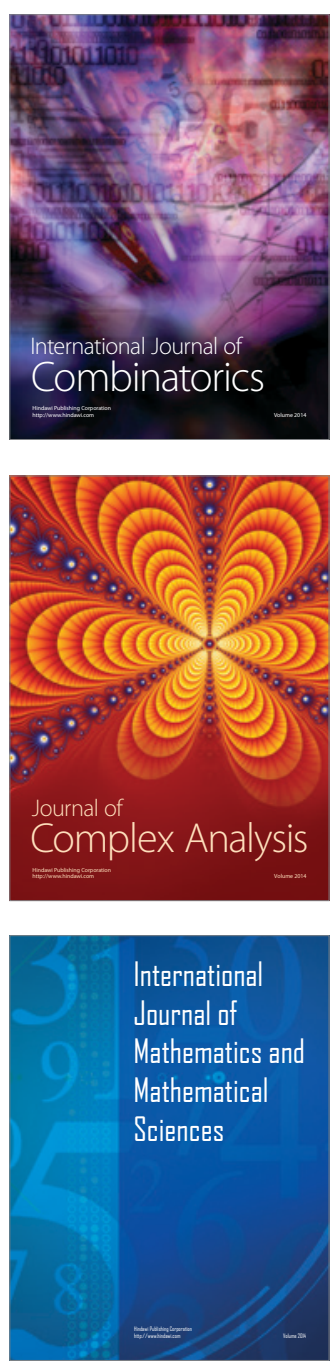
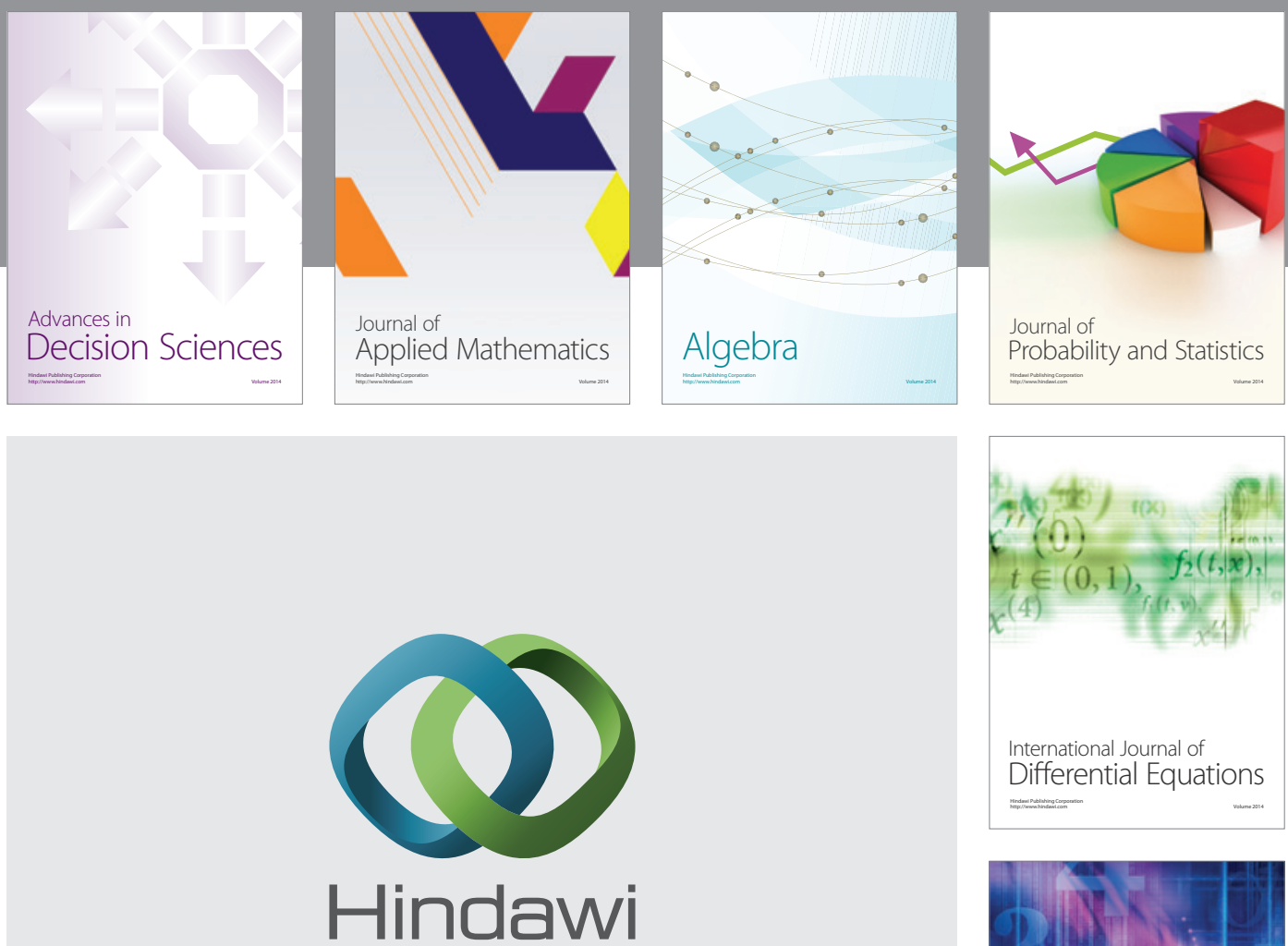

Submit your manuscripts at http://www.hindawi.com
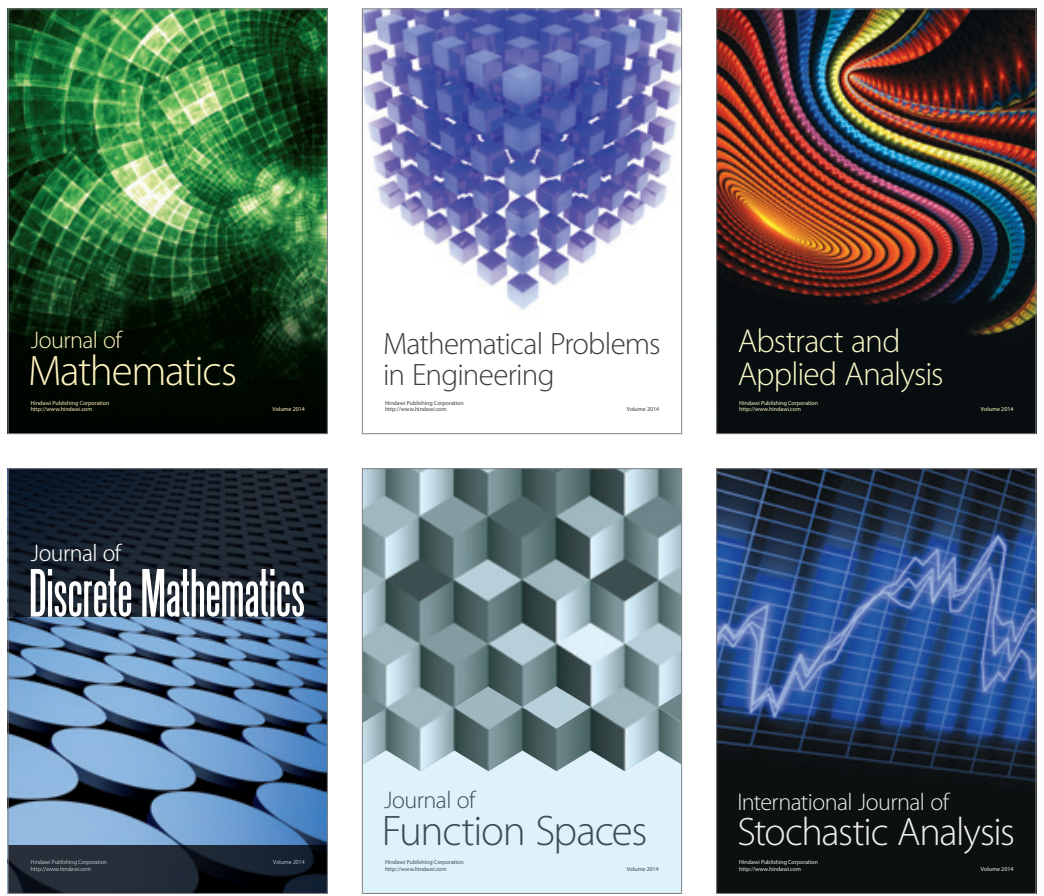

Journal of

Function Spaces

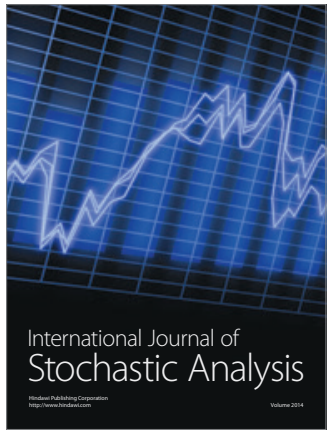

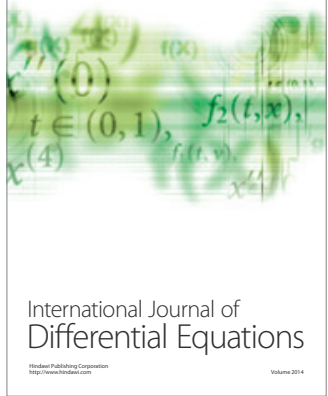
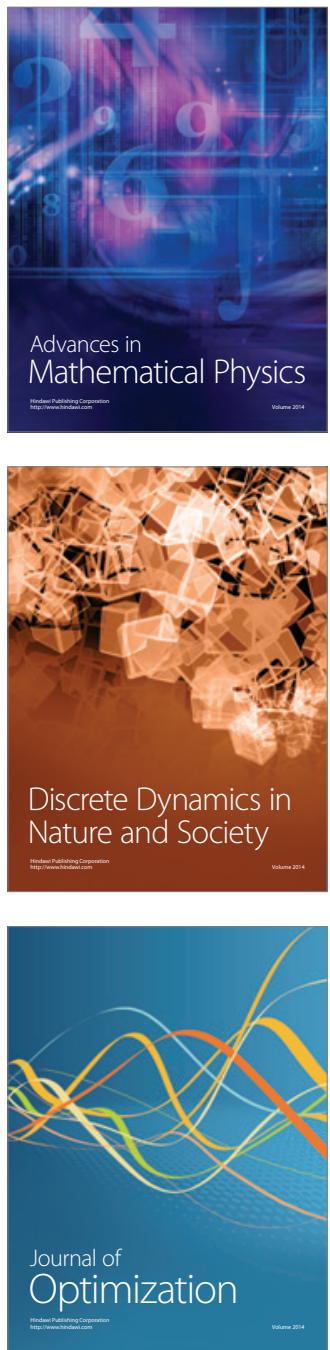